\title{
La curva de Kuznets ambiental y su relación con el cambio climático en América Latina y el Caribe: un análisis de cointegración con panel, 1980-2015
}

\author{
Recibido: julio, 17 de 2017 - Aprobado: febrero, 07 de 2019
}

Doi: http:/ /dx.doi.org/10.12804/revistas.urosario.edu.co/economia/a.7769

\author{
Luis Sánchez* \\ Karina Caballero ${ }^{\dagger}$
}

\section{Resumen}

Este artículo analiza la relación entre el cambio climático y la actividad económica en América Latina y el Caribe mediante la curva de Kuznets ambiental (CKA). Los resultados del modelo de panel con cointegración para las emisiones de $\mathrm{CO}_{2}$ indican la existencia de la CKA y la presencia de relaciones de cointegración entre las variables involucradas, por lo que es necesario alcanzar un punto de inflexión superior a 10.134 dólares per cápita (a precios de 2010) para poder estabilizar las emisiones y que, posteriormente, comiencen a reducirse, generando un proceso de actividad económica baja en carbono.

Palabras clave: curva de Kuznets ambiental, cambio climático, panel con cointegración, PIB per cápita, energía.

Clasificación JEL: Q43, Q42, C51, C53, E17.

* Facultad de Economía, Universidad Nacional Autónoma de México. Autor para correspondencia: Facultad de Economía, Universidad Nacional Autónoma de México (unam), Edificio de Posgrado, cub.18, Ciudad Universitaria, Ciudad de México, México. Correo electrónico: luiszbc@gmail.com. Teléfono: (+52 55) 5622-1888 ext. 48932.

+ Posgrado de la Facultad de Economía, Universidad Nacional Autónoma de México.

Para citar este artículo: Sánchez, L., \& Caballero, K. (2019). La Curva de Kuznets Ambiental y su relación con el cambio climático en América Latina y el Caribe: Un análisis de cointegración con panel, 1980-2015. Revista de Economía del Rosario, 22(1), 101-142. Dor: http:/ / dx.doi.org/10.12804/revistas.urosario.edu.co/economia/a.7769 


\title{
The Environmental Kuznets Curve and its Relationship with Climate Change in Latin America and the Caribbean: A Panel Cointegration Approach, 1980-2015
}

\begin{abstract}
This article analyses the relationship between climate change and economic activity in Latin America and the Caribbean through the environmental Kuznets curve (ЕКC). The results of the panel cointegration model for $\mathrm{CO}_{2}$ emissions indicates the existence of the EKC, the presence of cointegration relationships between the variables involved and the need to reach a break point greater than $\$ 10,134$ per capita (measured at 2010 prices) to stabilize emissions and subsequently begin to reduce generating a process of low carbon economic activity.
\end{abstract}

Keywords: Environmental Kuznets curve, climate change, panel cointegration, GDP per capita, energy.

JEL Classification: Q43, Q42, C51, C53, E17.

\section{A curva de Kuznets ambiental e sua relação com a mudança climática na América Latina e o Caribe: uma análise de cointegração com painel, 1980-2015}

\begin{abstract}
Resumo
Este artigo analisa a relação entre a mudança climática e a atividade econômica na América Latina e o Caribe mediante a curva de Kuznets ambiental (CKA). Os resultados do modelo de painel com cointegração para as emissões de $\mathrm{CO}_{2}$ indicam a existência da CKA, a presença de relações de cointegração entre as variáveis envolvidas e que é necessário alcançar um ponto de inflexão superior a 10,134 dólares per capita (a preços de 2010) para conseguir estabilizar as emissões e que, posteriormente, comecem a reduzir-se gerando um processo de atividade econômica baixa em carbono.

Palavras-chave: curva de Kuznets ambiental, mudança climático, painel com cointegração, РIв per capita, energia.

Classificação JEL: Q43, Q42, C51, C53, E17.
\end{abstract}




\section{Introducción}

El cambio climático se ha convertido en una amenaza para los seres vivos. Existe un consenso indicando que esta alteración climática es consecuencia de las altas emisiones y concentraciones de gases efectos invernadero (GEI) en la atmósfera, causados principalmente por las actividades antropogénicas como el uso de combustibles fósiles, así como por el cambio de suelo y la silvicultura. Una importante cantidad de estudios científicos confirman las alteraciones climáticas que se manifiestan constantemente a un ritmo acelerado.

Debido a la importancia de este problema global, y de no actuar, la temperatura terrestre seguirá incrementándose dificultando la vida en el planeta. En la región de América Latina y el Caribe (ALC) sus estructuras económicas han contribuido en el aumento de las emisiones de GEI, lo que hace necesario un análisis de las causas y los costos del cambio climático, aunque este sea una tarea compleja, pues es necesario basarse en diversas teorías y metodologías que logren una aproximación a la realidad, con el objetivo de proponer diversas políticas ambientales, económicas y sociales que ayuden a la adaptación de los impactos del cambio climático y a la mitigación de los GEI.

En consecuencia, el documento tiene los siguientes objetivos: identificar los factores y las magnitudes que determinan las emisiones de dióxido de carbono $\left(\mathrm{CO}_{2}\right)$ a nivel per cápita en 23 países de la región de ALC de 1980 a 2015; comprobar la existencia de la hipótesis de la curva de Kuznets ambiental (CKA), el cual es un análisis que favorece la búsqueda de propuestas de soluciones ambientales y económicas en los países pobres y en vía de desarrollo, además de permitir el cálculo del nivel de ingreso per cápita óptimo, que logre estabilizar las emisiones de $\mathrm{CO}_{2}$ y las disminuya, buscando contribuir con la descarbonización de las economías; y, finalmente, analizar y discutir algunas implicaciones de política pública y hacer algunas propuestas que podrían favorecer a los países para enfrentar los desafíos del cambio climático en las siguientes décadas.

El artículo se divide en cinco secciones. En la primera sección se presenta la importancia del fenómeno del cambio climático y sus impactos en la región de ALC, así como el análisis, en términos generales, del comportamiento económico regional y sus emisiones de $\mathrm{CO}_{2}$; la segunda sección muestra el marco teórico y la evidencia empírica sobre CKA; en la tercera sección, se presenta la metodología econométrica y los resultados de la estimación de un panel cointegrado basado en la CKA sobre las emisiones de $\mathrm{CO}_{2}$ per cápita para la región; finalmente, en la cuarta sección se presentan la discusión y el análisis de política pública. 


\section{América Latina y el Caribe: situación ante el cambio climático y su actividad económica}

El cambio climático es uno de los grandes desafíos de la humanidad en el siglo XxI. El Panel Intergubernamental del Cambio Climático (IPCC, por sus siglas en inglés) confirma que en las últimas décadas han ocurrido alteraciones climáticas globales por el aumento de las emisiones y concentraciones de GEI en la atmósfera -principalmente de $\mathrm{CO}_{2}-$, debido al uso intensivo de energía y al cambio de uso de suelo (IPCC, 2013). Las manifestaciones directas del cambio climático observables son el incremento en la temperatura, la alteración en los patrones de precipitación, la disminución de la criósfera y el aumento del nivel del mar y de la frecuencia e intensidad de los eventos extremos; estas manifestaciones inciden directamente en la actividad económica, en los ecosistemas, en la salud y en aspectos sociales (IPCC, 2007). La evidencia científica indica que la temperatura de la superficie terrestre y oceánica ha sido, en promedio, de $0,85^{\circ} \mathrm{C}$, considerando un rango entre $0,65^{\circ} \mathrm{C}$ y $1,06{ }^{\circ} \mathrm{C}$, desde 1880 hasta 2012 , destacando que durante el periodo comprendido entre 2003 y 2012, la temperatura ha tenido un incremento promedio de $0,78{ }^{\circ} \mathrm{C}$ con respecto al período entre 1850 y 1900. Las proyecciones en el aumento de temperatura para 2100 se establecen entre un rango de $1{ }^{\circ} \mathrm{C}$ a $3,7^{\circ} \mathrm{C}$, llegando incluso a niveles de $4,8^{\circ} \mathrm{C}$ en escenarios extremos, todo esto comparado con registros de la temperatura promedio del periodo 1850-1900 (IPCC, 2013). Las proyecciones de la temperatura promedio de la tierra están basadas en senderos de concentración representativa (RCP) los cuales destacan que a) el total de las emisiones acumuladas de $\mathrm{CO}_{2}$ y la temperatura de la superficie global tienen una relación lineal, por lo que el nivel de calentamiento está asociado a las emisiones acumuladas; b) una fracción del cambio climático antropogénico causado por las emisiones de $\mathrm{CO}_{2}$ es irreversible, a menos que se logre mitigar una cantidad importante de este gas en la atmósfera, debido a que la temperatura de la superficie terrestre se incrementará durante varios siglos, y c) es virtualmente probable que el nivel promedio del mar en el mundo aumentará entre uno y tres metros más allá del año 2100, como lo indica el escenario RCP8.5 (IPCC, 2013).

En ALC se han manifestado alteraciones en la temperatura que han sido registrados desde los años setenta, mostrando aumentos de $0,1^{\circ} \mathrm{C}$ en promedio para cada década. Se estima que hacia el 2100 -en términos del escenario más optimista de los RCP- la temperatura de la región aumente $1{ }^{\circ} \mathrm{C}$ y, en el escenario medio, se alcance un promedio de $2{ }^{\circ} \mathrm{C}$. Ambos escenarios son comparados con el período 1986-2005 (IPCC, 2013). Al mismo tiempo, existe gran incertidumbre en los patrones de precipitación, por lo que se proyectan 
cambios diferenciados en subregiones de ALC, así como la intensificación de eventos climáticos extremos (IPCC, 2013; PNUMA \& CEPAL, 2010). Los estudios realizados para la región indican que para finales del siglo XXI podría presentarse una mayor intensidad de los huracanes y una disminución en la precipitación acompañada de más sequías. En México habrá aumentos de temperatura, más ondas de calor, menos días con heladas y mayores sequías, mientras que en países como Ecuador, Colombia y Perú habrá diversas alteraciones en las precipitaciones, que traerán consigo efectos adversos sobre el rendimiento agrícola. A mediados del siglo xxI se pronostican amenazas en la disponibilidad de servicios ecosistémicos en la cordillera de los Andes, Centroamérica, el Caribe, México y en el suroriente de Brasil. También se pronostican efectos negativos en la pesca en el litoral pacífico del Perú y Chile, y con el aumento de la temperatura de la superficie de los océanos, se generará de forma más frecuente el blanqueamiento de los arrecifes de coral con efectos negativos para la pesca y el turismo; por otra parte, en Chile y Argentina los glaciares se verán afectados al disminuir su volumen (PNUMA \& CEPAL, 2010).

En el marco del desarrollo sustentable, el cambio climático ha acentuado los efectos negativos sobre el medio ambiente, en gran medida por el crecimiento económico, la producción y el consumo de energía y el cambio tecnológico (Goldemberg, Johansson, Reddy \& Williams, 1987). Sin embargo, existe una relación entre energía y el desarrollo sustentable, considerando un manejo adecuado y controlado, que puede llevar a cabo diversas acciones favorables, siendo la energía una parte importante en la discusión del desarrollo sustentable, puesto que es el núcleo en tres dimensiones: a) dimensión económica, donde la energía es el motor clave del crecimiento macroeconómico, porque contribuye a la transformación de insumos en bienes finales y servicios; b) dimensión social, que indica que el uso de energía se convierte en el principal prerrequisito que ayuda a satisfacer las necesidades humanas básicas (alimentación, salud, educación, vivienda y agua, principalmente), y c) dimensión ambiental, en que el uso de energéticos es la principal fuente de stress ambiental en todos los niveles; ésta relación entre la energía y el medio ambiente se determina por la extracción y producción de los combustibles y la calidad ambiental (Najam \& Cleveland, 2003; Munasinghe, 2002).

La región de ALC ha sido un abastecedor de recursos tanto naturales como elaborados tanto para su mercado interno, como para las principales economías del mundo; no obstante, la región ha sufrido inestabilidad económica durante las últimas tres décadas, la cual se ha reflejado en el nivel de vida de sus sociedades, en la disminución de la actividad económica, en 
el uso intensivo de energéticos de origen fósil y en el atraso tecnológico en relación con otros países.

El desempeño económico de ALC ha estado relacionado con la globalización, siendo un factor relevante que determina el desarrollo de los países y que se refleja en diversos aspectos como en la acumulación de capital, el progreso tecnológico, el comercio exterior, el comportamiento del Producto Interno Bruto (PIB), el empleo, la distribución de la riqueza y el ingreso, entre otros. Sin embargo, ante respuestas no satisfactorias en la aplicación de las políticas económicas, se han generado efectos que fracturan la realidad interna y obligan a decisiones que algunas veces están fuera del control de los mismos países (Ferrer, 1998).

En la tabla 1 se muestra que de 1980 a 2017 la región tuvo un crecimiento del PIB de 2,54\%, lo que se entiende como un reflejo de desaceleración de la actividad económica. Así mismo, el PIB per cápita de ALC presentó durante el mismo periodo un crecimiento promedio anual muy bajo de $0,97 \%$, lo que muestra que durante el periodo hubo importantes pérdidas en el poder adquisitivo y de calidad de vida de la población de la región. La energía, junto con factores como el trabajo, el capital y la tecnología, son necesarios para la producción y, en consecuencia, para el crecimiento económico (Stern \& Cleveland, 2003; Alam, 2006). Estas relaciones entre la energía y los factores productivos pueden transformar su rol en relación con el crecimiento de la economía, es decir, la energía y la producción se pueden modificar a través de la sustitución por otros insumos, por los cambios tecnológicos y por los cambios en su respectiva composición (Stern \& Cleveland, 2003). Por ende, en ALC hay presencia de tendencias ascendentes durante las últimas cuatro décadas en el consumo de energía, aunque muchos de esos países han tenido fluctuaciones importantes que han sido afectadas, principalmente por su respectiva estructura energética, así como por las crisis petroleras desde la década de los años setenta, las fuertes fluctuaciones en los precios de los energéticos, los cortes prolongados de abastecimiento eléctrico, el uso intensivo de energía y la dependencia en las importaciones de energéticos, entre otras situaciones (CEPAL, OLADE \& GTZ, 2003).

A pesar de las importantes fluctuaciones en el uso de energía en algunos países de la región, en ALC se ha registrado una tasa promedio anual de 2,32\% desde 1980 hasta 2015, destacando las altas tasas en Trinidad y Tobago, Chile, Bolivia, Ecuador, Guatemala y Panamá. En la mayoría de los países de la región el comportamiento del consumo de energía per cápita es ascendente, con excepción de Venezuela y aquellos países que pertenecen a las subregiones de Centroamérica y el Caribe como Belice, Jamaica, Cuba, Haití, Nicaragua y El Salvador. La disminución en el uso de energía per cá- 
pita puede ser resultado de la eficiencia de producción y abastecimiento del sector y de modificaciones en la población que provoca variaciones en los stocks de energéticos (tabla 1). Por otra parte, el uso de la energía renovable se ha vuelto más relevante en las actividades económicas de varios países, siendo considerada como una alternativa a las energías fósiles, debido a su disponibilidad y por su menor impacto sobre el medio ambiente; por consiguiente, en ALC de 1980 hasta 2015, el crecimiento promedio anual de este tipo de energéticos ha crecido en un $2,24 \%$, destacando su uso y producción en países como Brasil, Chile y Uruguay (tabla 1).

Tabla 1. Tasas de crecimiento medias anuales de variables macroeconómicas en los países de América Latina y el Caribe (en porcentajes)

\begin{tabular}{|c|c|c|c|c|c|c|}
\hline Región / País & PIB (\%) & $\begin{array}{l}\text { PIB per } \\
\text { cápita }(\%)\end{array}$ & $\begin{array}{c}\text { Consumo } \\
\text { de energía } \\
(\%)^{* *}\end{array}$ & $\begin{array}{l}\text { Consumo de } \\
\text { energía per } \\
\text { cápita }(\%)^{* * *}\end{array}$ & $\begin{array}{c}\text { Consumo de } \\
\text { energía reno- } \\
\text { vable (\%) }\end{array}$ & $\begin{array}{c}\text { Consumo de } \\
\text { energía renovable } \\
\text { per cápita }(\%)\end{array}$ \\
\hline $\begin{array}{l}\text { América } \\
\text { Latina y El } \\
\text { Caribe* }^{*}\end{array}$ & 2.54 & 0.97 & 2.32 & 0.72 & 2.24 & 0.64 \\
\hline Argentina & 1.94 & 0.69 & 2.01 & 0.76 & 2.45 & 1.19 \\
\hline Belice & 4.39 & 1.73 & 1.27 & -1.18 & 5.07 & 2.53 \\
\hline Bolivia & 3.04 & 1.16 & 3.55 & 1.61 & 0.73 & -1.16 \\
\hline Brasil & 2.22 & 0.72 & 2.61 & 1.06 & 2.33 & 0.79 \\
\hline Chile & 4.21 & 2.89 & 3.85 & 2.49 & 4.60 & 3.23 \\
\hline Colombia & 3.51 & 1.93 & 2.04 & 0.44 & 1.11 & -0.48 \\
\hline Costa Rica & 3.85 & 1.85 & 2.95 & 0.91 & 3.00 & 0.95 \\
\hline Cuba & 2.53 & 2.10 & -0.01 & -0.44 & -2.07 & -2.48 \\
\hline Ecuador & 2.99 & 0.81 & 3.29 & 1.23 & 2.26 & 0.22 \\
\hline El Salvador & 1.71 & 0.97 & 0.84 & -0.07 & -1.58 & -2.46 \\
\hline Guatemala & 2.92 & 0.60 & 3.25 & 0.89 & 3.25 & 0.88 \\
\hline Haití & 0.69 & -0.88 & 1.62 & -0.20 & 1.17 & -0.65 \\
\hline Honduras & 3.36 & 0.82 & 2.97 & 0.43 & 1.77 & -0.74 \\
\hline Jamaica & 1.52 & 0.72 & -0.20 & -1.01 & -1.64 & -2.43 \\
\hline México & 3.01 & 1.30 & 2.02 & 0.32 & 1.71 & 0.02 \\
\hline Nicaragua & 2.30 & 0.52 & 1.73 & -0.08 & 1.31 & -0.49 \\
\hline Panamá & 4.66 & 2.62 & 3.24 & 1.23 & 1.66 & -0.31 \\
\hline Paraguay & 3.47 & 1.36 & 2.66 & 0.52 & 2.39 & 0.26 \\
\hline Perú & 3.08 & 1.37 & 1.94 & 0.23 & -0.44 & -2.11 \\
\hline
\end{tabular}




\begin{tabular}{ccccccc}
\hline Región / País & PIB (\%) & $\begin{array}{c}\text { PIB per } \\
\text { cápita }(\%)\end{array}$ & $\begin{array}{c}\text { Consumo } \\
\text { de energía } \\
(\%)^{* *}\end{array}$ & $\begin{array}{c}\text { Consumo de } \\
\text { energía per } \\
\text { cápita }(\%)^{* *}\end{array}$ & $\begin{array}{c}\text { Consumo de } \\
\text { energía reno- } \\
\text { vable }(\%)\end{array}$ & $\begin{array}{c}\text { Consumo de } \\
\text { energía renovable } \\
\text { per cápita (\%) }\end{array}$ \\
\hline $\begin{array}{c}\text { República } \\
\text { Dominicana }\end{array}$ & 4.47 & 2.75 & 2.74 & 1.01 & -0.81 & -2.48 \\
$\begin{array}{c}\text { Trinidad y } \\
\text { Tobago }\end{array}$ & 1.97 & 1.33 & 6.39 & 5.71 & 3.53 & 2.86 \\
Uruguay & 2.29 & 1.83 & 2.19 & 1.72 & 4.41 & 3.93 \\
Venezuela & 1.18 & -0.79 & 1.74 & -0.25 & 5.25 & 3.19 \\
\hline
\end{tabular}

Periodos: PIв у PIв per cápita: 1980-2017, excepto Cuba y Venezuela (1980-2016) y Haití (1990 a 2017). Consumo de energía, consumo de energía per cápita, consumo de energía renovable y consumo de energía renovable per cápita: 1980-2015, excepto Belice (2001-2015).

* Se consideraron todos los países de América Latina y el Caribe.

** Se excluyó la energía renovable.

Fuente: elaboración propia con información de cepalstat de la Comisión Económica para América Latina y el Caribe (CEPAL) y del Sistema de Información Económica Energética de la Organización Latinoamericana de Energía (OLADE).

La intensidad energética es un indicador relevante que involucra al consumo de energía y al crecimiento económico de un país. En la tabla 2 se observa que de 1980 a 2015 hubo una importante dispersión entre los países, debido principalmente al uso diferenciado de los energéticos y a las estructuras productivas que utilizan una gran cantidad de energía.

Tabla 2. Tasas de crecimiento medias anuales de la intensidad energética en los países de América Latina y el Caribe (en porcentajes)

\begin{tabular}{cc}
\hline Región / País & Intensidad energética (\%) \\
\hline América Latina y El Caribe* & -0.33 \\
Argentina & -0.01 \\
Belice & -2.14 \\
Bolivia & 0.56 \\
Brasil & 0.18 \\
Chile & -0.50 \\
Colombia & -1.51 \\
Costa Rica & -0.87 \\
Cuba & -2.55 \\
Ecuador & 0.15 \\
El Salvador & -0.82
\end{tabular}




\begin{tabular}{cc}
\hline Región / País & Intensidad energética (\%) \\
\hline Guatemala & 0.33 \\
Haití & 1.72 \\
Honduras & -0.34 \\
Jamaica & -1.72 \\
México & -1.00 \\
Nicaragua & -0.42 \\
Panamá & -1.33 \\
Paraguay & -0.84 \\
Perú & -1.09 \\
República Dominicana & -1.60 \\
Trinidad y Tobago & 3.97 \\
Uruguay & -0.11 \\
Venezuela & 0.01 \\
\hline
\end{tabular}

Periodo: 1980-2015, excepto Belice (2001-2015) y Haití (1990 a 2015).

* se consideraron todos los países de América Latina y el Caribe. Se excluyó la energía renovable en el cálculo de la intensidad energética.

Fuente: elaboración propia con información de cepalstat de la Comisión Económica para América Latina y el Caribe (CEPAL) y del Sistema de Información Económica Energética (sIEE) de la Organización Latinoamericana de Energía (OLADE).

Datos recientes indican que ALC ha contribuido con cerca del 10\% de las emisiones de GEI a nivel mundial, básicamente por el crecimiento de la población y el uso de energéticos en el sector transporte (CEPAL, 2015; Sánchez \& Reyes, 2015; Sheinbaum \& Ruiz, 2012). Las trayectorias de las emisiones de $\mathrm{CO}_{2}$ totales son ascendentes, esto se refleja en tasas medias de crecimiento altas como en los casos de Trinidad y Tobago (6,32\%), Honduras (4,85\%) y Bolivia $(4,60 \%)$. Considerando a toda la región, la tasa de crecimiento promedio anual ha sido de 2,42\%. En el caso de las emisiones de $\mathrm{CO}_{2}$ per cápita, hay tendencias ascendentes y algunos países del Caribe y de Sudamérica registran los valores más altos de emisiones por habitante, lo que se manifiesta en aumentos progresivos en las emisiones (ver figura 1). 


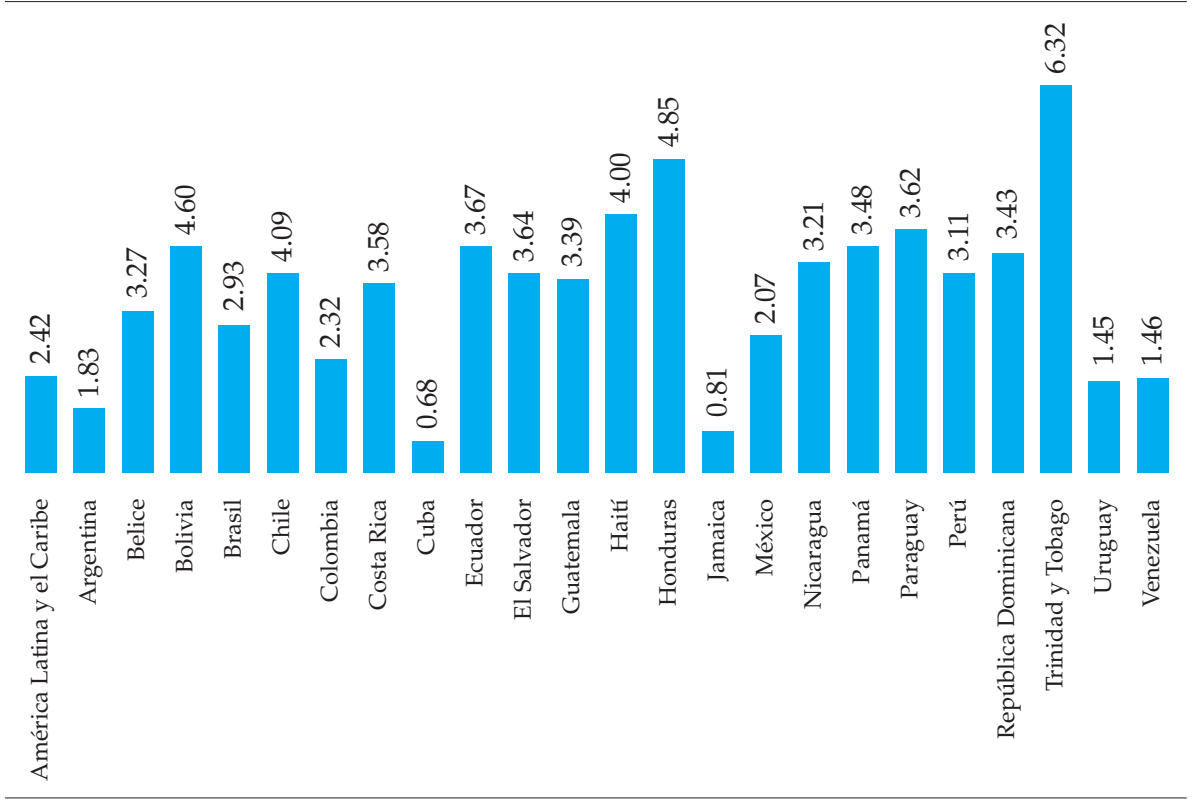

Emisiones de $\mathrm{CO}_{2}$ per cápita

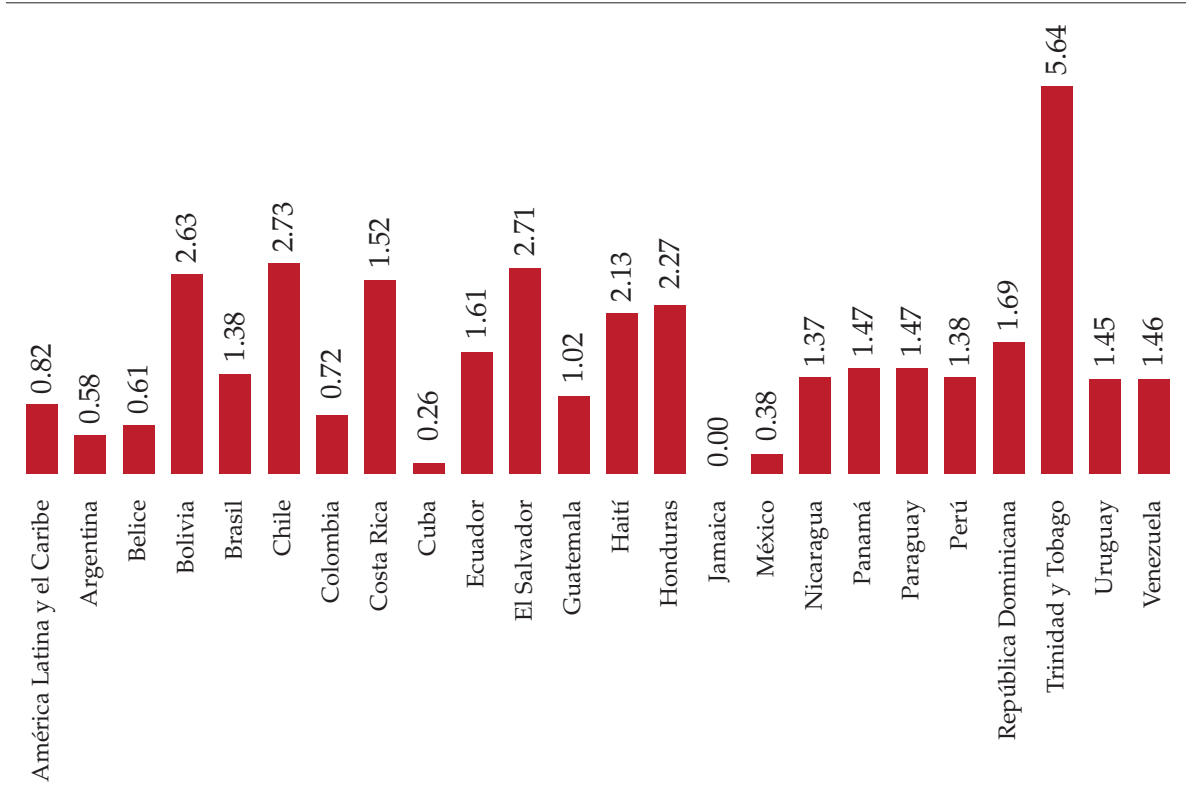

Figura 1. Tasas de crecimiento medias anuales de las emisiones de $\mathrm{CO}_{2}$ totales y per cápita en América Latina y el Caribe: 1980-2015 (En porcentajes)

Nota: Para América Latina y el Caribe se consideraron únicamente 23 países.

Fuente: elaboración propia con información obtenida de cepalstat de la Comisión Económica para América Latina y el Caribe (CEPAL). 
En cuanto a la relación entre emisiones de $\mathrm{CO}_{2}$ por unidad de PIB -denominado como intensidad carbónica o descarbonización- (figura 2), la trayectoria en ALC es decreciente, debido al constante aumento de las emisiones y a la evolución discreta de las economías; esta descarbonización registra una tasa de crecimiento promedio de $-0,23 \%$ anual durante el período comprendido entre 1980 y 2015. En las grandes economías de la región, como Argentina, Chile y México, la intensidad carbónica muestra un patrón similar comparado con el total de la región, es decir, con tendencias descendentes. Cabe señalar que esto podría ser una señal de que las políticas ambientales y económicas que se han estado implementando en la mayoría de los países de la región, han sido insuficientes para reducir la cantidad de emisiones de GEI. En cambio, en Brasil, el comportamiento de la descarbonización de su economía ha sido, en términos generales, positivo, a razón de su crecimiento económico hasta 2015 y el uso cada vez mayor de energías limpias.

\section{América Latina y El Caribe}

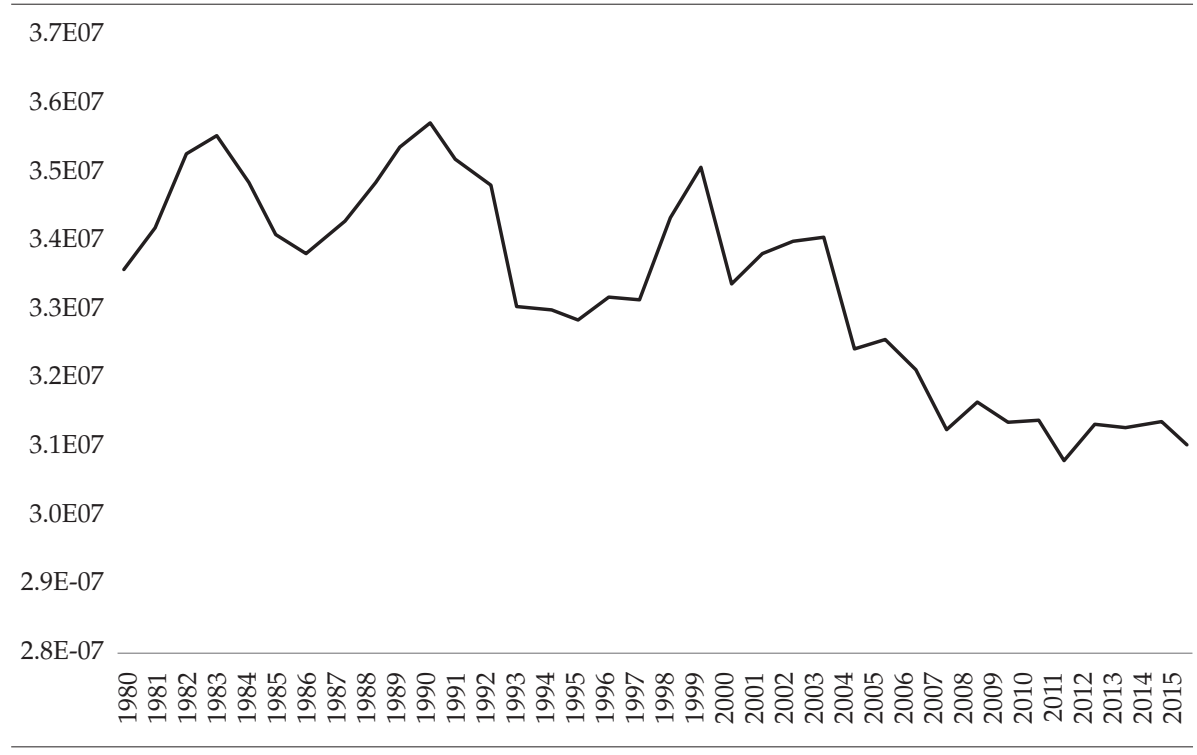

Continúa 


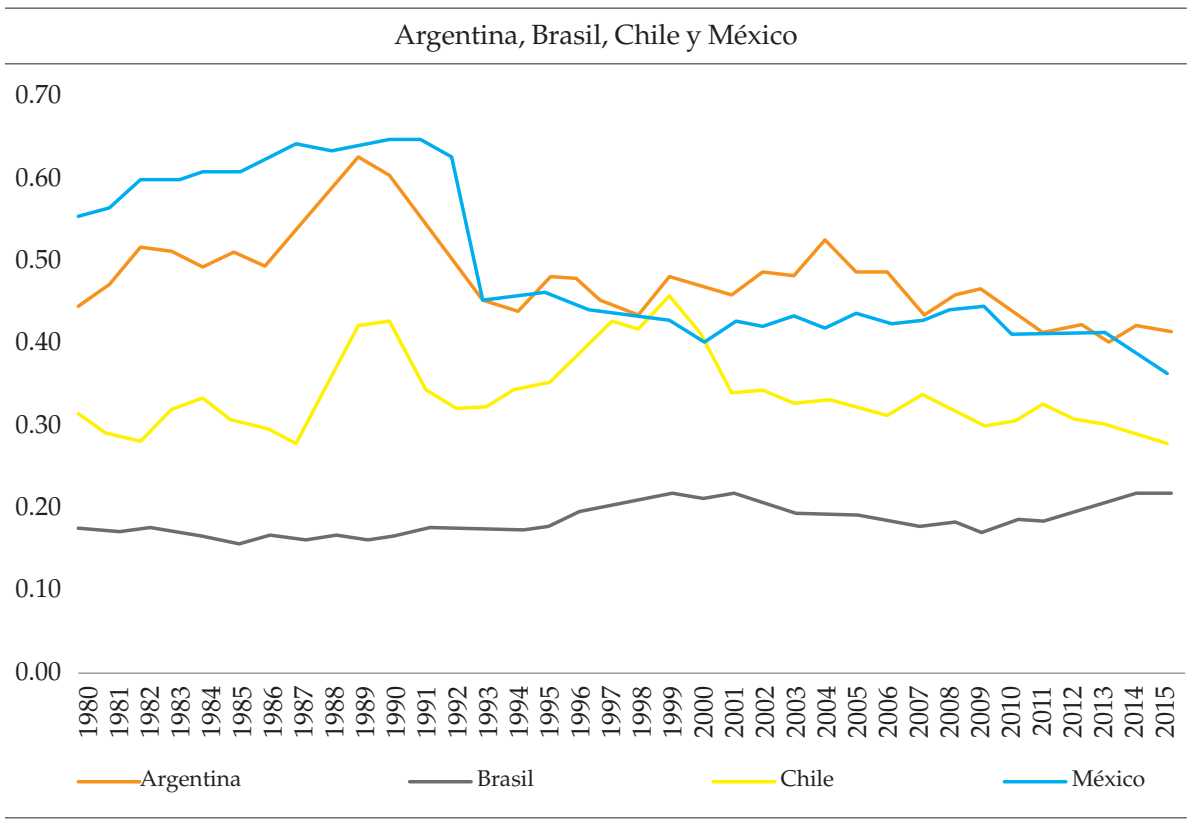

Figura 2. Trayectorias de las emisiones de $\mathrm{CO}_{2}$ por PІв (intensidad carbónica) en América Latina y el Caribe y países seleccionados: 1980-2015 (en miles de toneladas de $\mathrm{CO}_{2}$ por millones de US\$ a precios constantes de 2010)

Nota: Para América Latina y el Caribe se consideraron únicamente 23 países.

Fuente: elaboración propia con información obtenida de cEPALSTAT de la Comisión Económica para América Latina y el Caribe (CEPAL).

Las tasas de crecimiento promedio anuales de las emisiones de $\mathrm{CO}_{2}$ provenientes de la quema de combustibles fósiles han tenido incrementos importantes de 1992 a 2014 en Asia meridional (5,36\%), Asia oriental y el Pacífico $(4,74 \%)$, y Oriente Medio y el Norte de África (4,14\%) en comparación con la región de ALC (2,76\%). Se destaca el comportamiento negativo de Europa y Asia central (-0,91\%) donde, principalmente, los países europeos han aplicado medidas para controlar las emisiones de $\mathrm{CO}_{2}$ teniendo resultados notables. $\mathrm{La}$ tendencia del crecimiento de las emisiones en estas últimas regiones mencionadas se debe al comportamiento de los factores como la descarbonización, la intensidad energética y la intensidad carbónica del sector energía, donde las tasas de crecimiento respectivas muestran comportamientos más intensos en comparación con otras regiones (figura 3). 


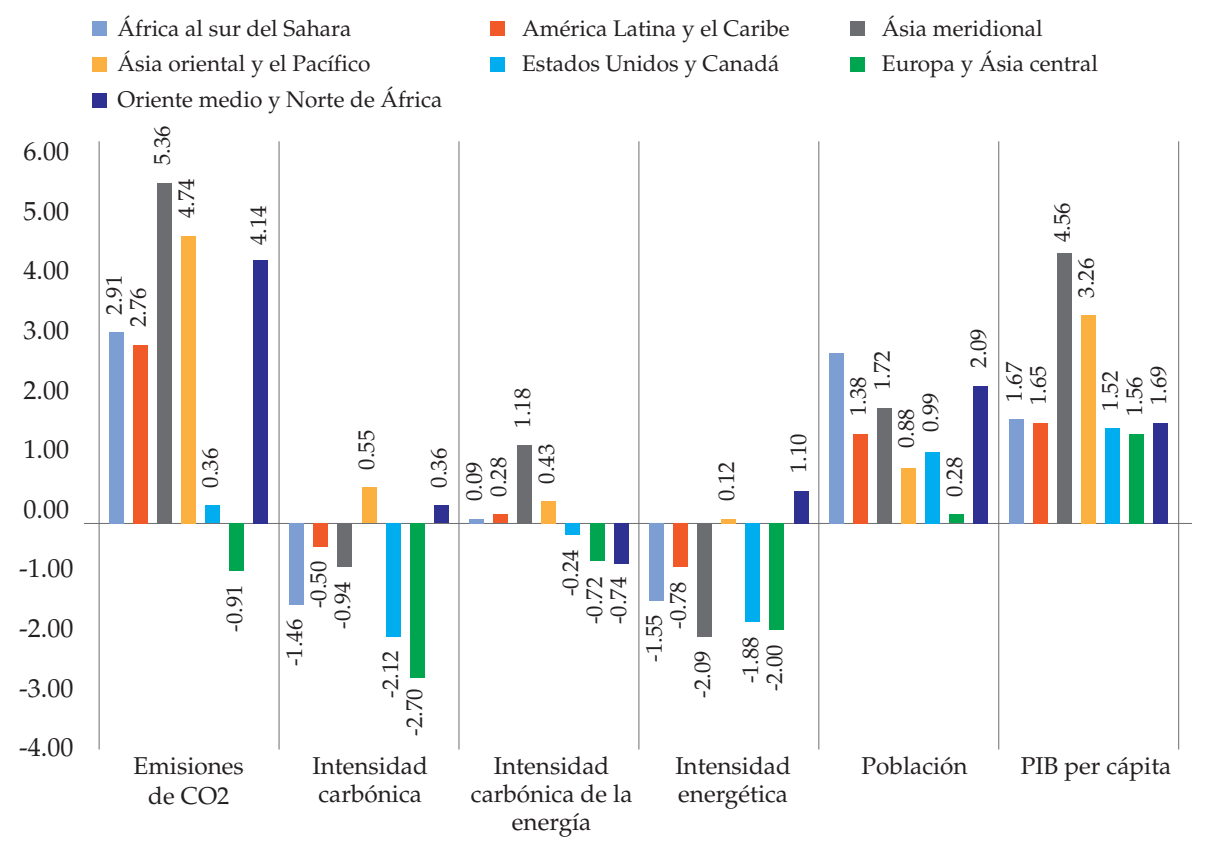

Figura 3. Tasas de crecimiento medias anuales de las emisiones de $\mathrm{CO}_{2} \mathrm{y}$ principales factores por regiones: 1992-2014 (en porcentajes)

Notas: Las emisiones de $\mathrm{CO}_{2}$ se refieren únicamente a la provenientes de la quema de combustibles fósiles y de la fabricación del cemento. Incluyen el dióxido de carbono producido durante el consumo de combustibles sólidos, líquidos, gaseosos y de la quema de gas.

Fuente: elaboración propia con información del Banco Mundial.

\section{Marco teórico y evidencia de la curva de Kuznets ambiental}

Es conocido que existen limitantes naturales al crecimiento económico y que, al mantenerse un determinado ritmo de crecimiento, se tendrán repercusiones sobre insumos estratégicos, el aumento de la contaminación ambiental y el crecimiento descontrolado de la población, lo que pone en riesgo el abastecimiento y la sustentabilidad en el futuro (Meadows, Meadows, Randers \& Behrens, 1972). Esta relación entre economía y degradación ambiental ha permitido el surgimiento de diversas teorías, entra entre las que destaca la hipótesis de la curva de Kuznets ambiental (CKA), que propone que a medida de que existe crecimiento económico, el impacto o daño ambiental tenderá a incrementarse en una primera fase y posteriormente llegará a un equilibrio y disminuirá (Ekins, 2015; Common \& Stagl, 2008; Labandeira, León \& Vásquez, 2007). El fundamento que se encuentra detrás de esta teoría señala que en una economía en crecimiento, la relación entre la desigualdad de los 
ingresos y el ingreso per cápita promedio presentan una forma de una curva $U$ invertida, es decir, existe una relación funcional cóncava; esto significa que en ingresos per cápita bajos, la desigualdad va a aumentar a la par, pero superado determinado nivel de ingreso, la desigualdad tenderá a disminuir (Kuznets, 1955; 1965).

Desde el punto de vista ambiental, tanto en la oferta como en la demanda existe una presión en la utilización de materias primas y energéticos, principalmente los de origen fósil, obligando a una mayor degradación y contaminación ambiental hasta llegar a un punto de inflexión a causa de haber conseguido un significativo proceso de industrialización. Por lo tanto, la hipótesis de la CKA ayudaría como referente para soluciones ambientales y económicas en los países pobres y en desarrollo, mediante el diseño de instrumentos que mejoren el medio ambiente y protejan los recursos naturales. Las acciones por tomar deben considerar regulaciones más estrictas, el desarrollo de mejores tecnologías, la modificación del crecimiento económico de los sectores más contaminantes y la modificación de los patrones de consumo (Kolstad, 2001; Molina \& Molina, 2005; Sánchez, 2013). Sin embargo, es relevante considerar que muchos países con ingresos muy bajos tienen como prioridad la satisfacción de necesidades básicas como la alimentación, el vestido y calzado y la vivienda (Sánchez, 2013).

El comienzo de la CKA parte de un modelo donde la economía es perfectamente competitiva y en el siguiente escenario se consideran las imperfecciones del mercado. De esta manera, los agentes (consumidor o empresa) maximizan sus beneficios netos $(B L)$, considerando que los beneficios $(B)$ y los costos $(C)$ son dependientes del nivel de ingreso per cápita $(Y)$ y de la calidad ambiental (E) -también denominada degradación ambiental-como se representa en la ecuación 1 (Munasinghe, 1999; Granja; Mendonça \& Nogueira, 2002):

$$
\operatorname{Max} B L=B(E, Y)-(E, Y)
$$

En consecuencia, en cualquier nivel de ingreso los individuos desean maximizar $B L$ a un valor donde los beneficios marginales $(B M)$ o la voluntad a pagar cierta calidad ambiental es igual al costo marginal $(C M)$. En el equilibrio, la degradación ambiental es optimizada por un determinado nivel de ingreso, e incrementa cuando el ingreso per cápita aumenta, de acuerdo con el modelo de la $U$ invertida, cambiando el efecto de positivo a negativo en un determinado nivel de ingreso. El comportamiento de la condición de optimalidad de $B M$ que es igual al $C M$ determinará el comportamiento de la CKA, donde se presentará un efecto positivo en escenarios iniciales de desarrollo económico pero que se convertiría en negativo después de que el nivel 
de ingreso sea mayor. En la parte descendente de la CKA, el BM se mueve ascendente ante las tasas de crecimiento del ingreso, por lo que la gente apreciará más los bienes y servicios ambientales y tendrán una mayor voluntad a pagar por ellos conforme incrementa su ingreso. Cuando la curva de CM es baja a causa de la degradación y el bajo nivel de desarrollo económico, su función no es relevante; no obstante, los CM de la protección ambiental se incrementarán rápidamente, tan pronto como la economía se desarrolle y crezca el ingreso al superar escenarios iniciales de desarrollo industrial e incrementos en los niveles de contaminación. Estos efectos ocurren en la fase de desarrollo económico asociado con el uso de recursos naturales y la generación de contaminación. Estas externalidades negativas pueden ser mitigadas con políticas ambientales y económicas junto con el uso de conocimiento y tecnología. Investigaciones como las de Munasinghe (1999) y Granja et al (2002) sugieren que el efecto descendente de la CKA se puede presentarse mediante: a) el efecto tecnología, donde a través de recursos humanos y el mejoramiento de la ciencia y la tecnología se reducen marginalmente el abatimiento y los costos protegidos con altos niveles de ingreso; y b) el efecto composición, que se puede relacionar con los cambios estructurales de la economía, siendo el sector servicios un elemento importante en la composición del PIB. En el escenario de equilibrio competitivo, el óptimo de Pareto garantiza que el nivel de degradación ambiental sea socialmente deseable en la curva completa; sin embargo, es posible identificar situaciones donde las imperfecciones de la economía proporcionan un patrón que no sea óptimo o económicamente ineficiente. Entonces, las curvas BM y $C M$, basadas en las decisiones de los consumidores y productores, podrían diferir de la curva óptima social resultando un nivel de degradación más alto que lo socialmente deseable.

De acuerdo con el modelo planteado por Kuznets (1955; 1965), se han hecho modificaciones en la especificación original a causa de la adaptación a casos ambientales, planteando una ecuación del siguiente tipo (Ekins, 2000):

$$
f\left(E_{i t}\right)=\alpha_{0}+\alpha_{1} g_{1}\left(Y_{i t}\right)+\alpha_{2} g_{2}\left(Y_{i t}^{2}\right)+\alpha_{3} g_{3}\left(Y_{i t}^{3}\right)+\alpha_{4} g_{4}\left(Y_{i t-a}^{n}\right)+\beta \cdot B+\gamma r+\varepsilon_{i t}
$$

Donde $\left(E_{i t}\right)$ representa un indicador ambiental de un país o región $i$ en el tiempo $t ; \alpha, \beta$ y $\gamma$ son los parámetros que deben ser estimados; $Y_{i t}$ es el ingreso per cápita de un país o región $i$ en el tiempo $t ; Y_{i t-a}{ }^{n}$ es un término polinomial de ingreso per cápita; $B$ es un vector de que representa otras variables explicativas que pueden incluir diversas variables económicas y / o de tipo dummy para capturar las influencias especificas de demografía, geografía o determinados años; en último lugar $f($.$) y g($.) son las formas funcionales que son 
predominantes pero no exclusivamente de tipo logaritmo o lineal. La forma funcional de la CKA debe ser cuadrática con los siguientes parámetros: $\alpha_{1}>0$, $\alpha_{2}<0, \alpha_{3}=0 ;\left|\alpha_{2}\right|<<\left|\alpha_{1}\right|$. Así mismo, el punto de inflexión per cápita del ingreso $(\varphi)$ representará el indicador ambiental que es maximizado mediante el siguiente calculo: $\varphi=-\frac{\alpha_{1}}{2 \alpha_{2}}$.

La CKA es un tema relevante que ha sido estudiado frecuentemente para comprobar su existencia. En la tabla 3 se presentan de manera resumida algunas de las investigaciones llevadas a cabo. Esta evidencia servirá para la identificación de variables relevantes que pueden estar involucradas en la determinación de la cKA para ALC.

Tabla 3. Resumen de la evidencia empírica sobre la curva de Kuznets ambiental

\begin{tabular}{|c|c|c|c|c|c|}
\hline Autores & $\begin{array}{l}\text { Lugar y } \\
\text { periodo }\end{array}$ & $\begin{array}{l}\text { Contaminantes* } \\
\text { (Variables endó- } \\
\text { genas en térmi- } \\
\text { nos per cápita) }\end{array}$ & $\begin{array}{l}\text { Variables } \\
\text { exógenas }\end{array}$ & $\begin{array}{l}\text { Métodología } \\
\text { econométrica }\end{array}$ & $\begin{array}{c}\text { Evidencia de } \\
\text { CKA-Punto de in- } \\
\text { flexión (promedio } \\
\text { o rango) }\end{array}$ \\
\hline $\begin{array}{l}\text { Grossman } \\
\text { y Krueger } \\
(1991)\end{array}$ & $\begin{array}{l}52 \text { ciudades } \\
\text { de } 32 \text { países } \\
(1977,1982 \text { y } \\
1988)\end{array}$ & $\begin{array}{l}\text { Concentracio- } \\
\text { nes de } \mathrm{SO}_{2} \text {, hu- } \\
\text { mo fino y SPM }\end{array}$ & $\begin{array}{l}\text { Рів per cápita, } \\
\text { densidad de po- } \\
\text { blación, variables } \\
\text { dummies locales } \\
\text { y tendencia }\end{array}$ & Panel & $\$ 7,132-8,915$ dls \\
\hline $\begin{array}{l}\text { Panayotou } \\
(1993)\end{array}$ & $\begin{array}{l}55 \text { países de- } \\
\text { sarrollados y } \\
\text { en desarrollo } \\
(1987-1988)\end{array}$ & $\begin{array}{l}\text { Emisiones de } \\
\mathrm{SO}_{2}, \mathrm{NOx}, \mathrm{SPM} \\
\text { y tasa de desa- } \\
\text { forestación }\end{array}$ & $\begin{array}{l}\text { PIB per cápita, } \\
\text { densidad de la } \\
\text { población y va- } \\
\text { riables dummies } \\
\text { para los países } \\
\text { tropicales }\end{array}$ & Panel & $\begin{array}{l}\mathrm{SO}_{2}: \$ 5,444 \text { dls., } \\
\mathrm{NOx}: \$ 9,981 \text { dls., } \\
\text { SPM: } \$ 8,166 \text { dls. } \\
\text { y deforestación: } \\
\$ 1,494 \text { dls. }\end{array}$ \\
\hline $\begin{array}{l}\text { Selden } \\
\text { y Song } \\
(1994)\end{array}$ & $\begin{array}{l}30 \text { países } \\
(1973-1975, \\
1979-1981 \text { y } \\
1982-1984)\end{array}$ & $\begin{array}{l}\text { Emisiones de } \\
\mathrm{SO}_{2}, \mathrm{NOx}, \mathrm{SPM} \\
\text { y } \mathrm{CO}\end{array}$ & $\begin{array}{l}\text { РІв per cápita y } \\
\text { densidad de la } \\
\text { población }\end{array}$ & Panel & $\begin{array}{l}\mathrm{SO}_{2}: \$ 11,473 \\
\text { dls., NOx: } \$ \\
14,777 \text { dls., sPM: } \\
\$ 13,554 \text { dls. y } \\
\text { CO: } \$ 7,855 \text { dls. }\end{array}$ \\
\hline $\begin{array}{l}\text { Cole et al } \\
(1997)\end{array}$ & $\begin{array}{l}11 \text { países de } \\
\text { la ocDE (1970- } \\
1992)\end{array}$ & $\begin{array}{l}\text { Emisiones de } \\
\mathrm{SO}_{2} .\end{array}$ & $\begin{array}{l}\text { PIB per cápita, va- } \\
\text { riables dummies } \\
\text { por país y nivel } \\
\text { de tecnología. }\end{array}$ & Panel & $\$ 22,014$ dls. \\
\hline $\begin{array}{l}\text { Kauffman } \\
\text { et al (1998) }\end{array}$ & $\begin{array}{l}13 \text { países desa- } \\
\text { rrollados y } 10 \\
\text { en desarrollo } \\
(1974-1989)\end{array}$ & $\begin{array}{l}\text { Concentracio- } \\
\text { nes de } \mathrm{SO}_{2}\end{array}$ & $\begin{array}{l}\text { РІB per cápita, } \\
\text { PIB /área y expor- } \\
\text { taciones de hierro } \\
\text { y acero/área }\end{array}$ & $\begin{array}{l}\text { Sección cruza- } \\
\text { da y Panel }\end{array}$ & $\begin{array}{l}\$ 5,444-22,684 \\
\text { dls. }\end{array}$ \\
\hline $\begin{array}{l}\text { Stern y } \\
\text { Common } \\
(2001)\end{array}$ & $\begin{array}{l}73 \text { países de- } \\
\text { sarrollados y } \\
\text { en desarrollo } \\
(1960-1990)\end{array}$ & $\begin{array}{l}\text { Emisiones de } \\
\mathrm{SO}_{2}\end{array}$ & $\begin{array}{l}\text { PIв per cápita, } \\
\text { tendencia y efec- } \\
\text { tos por país }\end{array}$ & Panel & $\$ 151,192$ dls. \\
\hline
\end{tabular}




\begin{tabular}{|c|c|c|c|c|c|}
\hline Autores & $\begin{array}{l}\text { Lugar y } \\
\text { periodo }\end{array}$ & $\begin{array}{l}\text { Contaminantes* } \\
\text { (Variables endó- } \\
\text { genas en térmi- } \\
\text { nos per cápita) }\end{array}$ & $\begin{array}{l}\text { Variables } \\
\text { exógenas }\end{array}$ & $\begin{array}{l}\text { Métodología } \\
\text { econométrica }\end{array}$ & $\begin{array}{c}\text { Evidencia de } \\
\text { CKA-Punto de in- } \\
\text { flexión (promedio } \\
\text { o rango) }\end{array}$ \\
\hline
\end{tabular}

Anderson China e India y Caven- (nd) y países dish (2001) en desarrollo

Emisiones de $\mathrm{SO}_{2}$ y $\mathrm{CO}_{2}$
PIB per cápita MCO

del aire Chile (nd)

Países de la

PIB per cápita y uso de energía per cápita comercial

PIB per cápita cial)
Galeotti et al (2006) OCDE (19501997)

$\begin{array}{ll}\text { Maddison } & 135 \text { países } \\ \text { (2006) } & (1990 \text { y 1995) }\end{array}$

Ang (2007) Francia (19602000)

Li et al 141 estudios (2007) (1992-2005)

\section{Emisiones de $\mathrm{CO}_{2}$} Emisiones de
$\mathrm{SO}_{2}, \mathrm{NOx}, \mathrm{VOC}$
y $\mathrm{CO}$

\section{Emisiones de $\mathrm{CO}_{2}$}

Variable tricotómica entre la relación medio ambiente- ingreso

Costa Rica, El Salvador, GuaApergis y Payne (2009) temala, Honduras, Nicaragua y Panamá (1971-2004)

Itkonen Fracia (1960(2010)

\section{Emisiones de \\ $\mathrm{CO}_{2}$}

PIB per cápita y variables de progreso técnico
Modelos de simulación dinámica
PIB per cápita y uso de energía per cápita

Meta-análisis

$\$ 37,217$ dls.

Panel (econometría espa-

China $\left(\mathrm{SO}_{2}\right)$ : $\$ 5,515$ dls. India $\left(\mathrm{SO}_{2}\right)$ : $\$ 3,309$ dls. Países en desarrollo $\left(\mathrm{CO}_{2}\right)$ : $\$ 5,515$ dls.

Existe evidencia de CKA y se pronostican reducciones del $50 \%$ en los contaminantes durante la proxima década

$\$ 17,366$ dls.

Hay evidencia de CKA para cada país pero no se calculan puntos de inflexión

Hay evidencia Cointegración de cKA pero no (Johansen) se calcula punto de inflexión

Panel coin- Hay evidencia tegrado de CKA para ca(FMOLs-Fully da país pero no Modified oLs) se calcula puny MCE tos de inflexión

Hay evidencia

PIB per cápita y uso de energía
Emisiones de $\mathrm{CO}_{2}$ $\begin{array}{ll}\text { Cointegración } & \text { de CKA pero no } \\ \text { (Johansen) } & \text { se calcula punto }\end{array}$ de inflexión 


\begin{tabular}{|c|c|c|c|c|c|}
\hline Autores & $\begin{array}{l}\text { Lugar y } \\
\text { periodo }\end{array}$ & $\begin{array}{l}\text { Contaminantes* } \\
\text { (Variables endó- } \\
\text { genas en térmi- } \\
\text { nos per cápita) }\end{array}$ & $\begin{array}{l}\text { Variables } \\
\text { exógenas }\end{array}$ & $\begin{array}{l}\text { Métodología } \\
\text { econométrica }\end{array}$ & $\begin{array}{c}\text { Evidencia de } \\
\text { CKA-Punto de in- } \\
\text { flexión (promedio } \\
\text { o rango) }\end{array}$ \\
\hline Xu (2012) & $\begin{array}{l}\text { China (1980- } \\
\text { 2008) }\end{array}$ & $\begin{array}{l}\text { Emisiones de } \\
\mathrm{CO}_{2}\end{array}$ & $\begin{array}{l}\text { РІв per cápita, } \\
\text { consumo de } \\
\text { energía per cápi- } \\
\text { ta y ratio de aper- } \\
\text { tura comercial }\end{array}$ & $\begin{array}{l}\text { Cointegración } \\
\text { (Engle-Gran- } \\
\text { ger) }\end{array}$ & $\$ 4,341$ dls. \\
\hline $\begin{array}{l}\text { Goldman } \\
(2012)\end{array}$ & $\begin{array}{l}120 \text { estudios } \\
(1992-2012)\end{array}$ & $\begin{array}{l}\text { Variable trico- } \\
\text { tómica entre la } \\
\text { relación medio } \\
\text { ambiente-in- } \\
\text { greso }\end{array}$ & PIB per cápita & $\begin{array}{l}\text { Meta-análi- } \\
\text { sism (Modelo } \\
\text { logit multino- } \\
\text { mial) }\end{array}$ & $\begin{array}{l}\text { Hay evidencia } \\
\text { de cKA pero no } \\
\text { se calcula punto } \\
\text { de inflexión }\end{array}$ \\
\hline $\begin{array}{l}\text { Fan y } \\
\text { Zheng } \\
(2013)\end{array}$ & $\begin{array}{l}\text { Provincia } \\
\text { Sichuan, China } \\
(1985-2010)\end{array}$ & $\begin{array}{l}\text { Emisiones in- } \\
\text { dustriales }\end{array}$ & PIB per cápita & $\mathrm{MCO}$ & $\begin{array}{l}\text { Hay evidencia } \\
\text { de cKA pero no } \\
\text { se calcula punto } \\
\text { de inflexión }\end{array}$ \\
\hline $\begin{array}{l}\text { Al Sayed y } \\
\text { Sek (2013) }\end{array}$ & $\begin{array}{l}40 \text { países cla- } \\
\text { sificados en } \\
\text { desarrollados } \\
\text { y en desarrollo } \\
(1961-2009)\end{array}$ & $\begin{array}{l}\text { Emisiones de } \\
\mathrm{CO}_{2}, \mathrm{SO}_{2}, \text { BOD, } \\
\text { SPM y GEI }\end{array}$ & РIB per cápita & Panel & $\begin{array}{l}\text { Países desa- } \\
\text { rrollados: } \mathrm{CO}_{2}: \\
41,368.49 \text { dls., } \\
\mathrm{SO}_{2}: 3,477.73 \\
\text { dls., BOD: } \\
\text { 18,840.24 dls y } \\
\text { GEI: } 7,690.7 \text { dls. } \\
\text { Países en desa- } \\
\text { rrollo: } \mathrm{CO}_{2} \text { : } \\
6,196.54 \mathrm{dls}, \mathrm{SO}_{2} \text { : } \\
44298.86 \mathrm{dls} ., \\
\text { BOD: } 6,404.18 \text { dls } \\
\text { y GEI: } 1,748.3 \mathrm{dls} \text {. }\end{array}$ \\
\hline $\begin{array}{l}\text { Zilio y } \\
\text { Caraballo } \\
(2014)\end{array}$ & $\begin{array}{l}21 \text { países de } \\
\text { América Lati- } \\
\text { na y el Caribe } \\
(1960-2008)\end{array}$ & $\begin{array}{l}\text { Emisiones de } \\
\mathrm{CO}_{2}\end{array}$ & $\begin{array}{l}\text { PIB per cápita, } \\
\text { término autorre- } \\
\text { gresivo del CO2, } \\
\text { valor agregado } \\
\text { de la industria, } \\
\text { importaciones } \\
\text { netas de energía } \\
\text { y apertura co- } \\
\text { mercial }\end{array}$ & $\begin{array}{l}\text { Modelos } \\
\text { semiparamé- } \\
\text { tricos }\end{array}$ & $\begin{array}{l}\text { No hay eviden- } \\
\text { cia de CKA }\end{array}$ \\
\hline $\begin{array}{l}\text { Cho et al } \\
(2014)\end{array}$ & $\begin{array}{l}\text { Países de la } \\
\text { ocDE }\end{array}$ & $\begin{array}{l}\text { Emisiones de } \\
\mathrm{CO}_{2}, \mathrm{~N}_{2} \mathrm{O} \text { y CH}\end{array}$ & $\begin{array}{l}\text { PIB per cápita, } \\
\text { consumo de } \\
\text { energía per cá- } \\
\text { pita }\end{array}$ & $\begin{array}{l}\text { Panel cointe- } \\
\text { grado } \\
\text { (FMoLs-Fully } \\
\text { Modified oLs) }\end{array}$ & $\begin{array}{l}\text { Hay evidencia } \\
\text { de cKA pero no } \\
\text { se calculan pun- } \\
\text { tos de inflexión }\end{array}$ \\
\hline $\begin{array}{l}\text { Farhani } \\
\text { et al (2014) }\end{array}$ & $\begin{array}{l}10 \text { países de } \\
\text { oriente medio } \\
\text { y África del } \\
\text { norte (1990- } \\
2010)\end{array}$ & $\begin{array}{l}\text { Emisiones de } \\
\mathrm{CO}_{2}\end{array}$ & $\begin{array}{l}\text { PIB per cápita, } \\
\text { consumo de ener- } \\
\text { gía, valor agrega- } \\
\text { do de la manufac- } \\
\text { tura; apertura } \\
\text { comercial e indice } \\
\text { de desarrollo hu- } \\
\text { mano }\end{array}$ & $\begin{array}{l}\text { Panel cointe- } \\
\text { grado (FMOLS- } \\
\text { Fully Modi- } \\
\text { fied oLs) }\end{array}$ & $\begin{array}{l}\text { Hay evidencia } \\
\text { de cKA pero no } \\
\text { se calculan pun- } \\
\text { tos de inflexión }\end{array}$ \\
\hline
\end{tabular}




\begin{tabular}{|c|c|c|c|c|c|}
\hline Autores & $\begin{array}{l}\text { Lugar y } \\
\text { periodo }\end{array}$ & $\begin{array}{l}\text { Contaminantes* } \\
\text { (Variables endó- } \\
\text { genas en térmi- } \\
\text { nos per cápita) }\end{array}$ & $\begin{array}{l}\text { Variables } \\
\text { exógenas }\end{array}$ & $\begin{array}{l}\text { Métodología } \\
\text { econométrica }\end{array}$ & $\begin{array}{c}\text { Evidencia de } \\
\text { CKA-Punto de in- } \\
\text { flexión (promedio } \\
\text { o rango) }\end{array}$ \\
\hline $\begin{array}{l}\text { Yang et al } \\
\text { (2015) }\end{array}$ & $\begin{array}{l}67 \text { países } \\
(1971-2010)\end{array}$ & $\begin{array}{l}\text { Emisiones de } \\
\mathrm{CO}_{2}\end{array}$ & PIB per cápita & $\begin{array}{l}\text { Método de } \\
\text { regresión sim- } \\
\text { bólica }\end{array}$ & $\begin{array}{l}\text { Existe eviden- } \\
\text { cia de la CKA en } \\
\text { algunos países, } \\
\text { pero, en general, } \\
\text { el modelo de } \\
\text { tipo N invertida } \\
\text { es más significa- } \\
\text { tivo en general }\end{array}$ \\
\hline $\begin{array}{l}\text { Yin et al } \\
(2015)\end{array}$ & $\begin{array}{l}\text { China (1999- } \\
\text { 2011) }\end{array}$ & $\begin{array}{l}\text { Emisiones de } \\
\mathrm{CO}_{2}\end{array}$ & $\begin{array}{l}\text { PIB per cápita, } \\
\text { consumo de } \\
\text { energía y progre- } \\
\text { so técnico, estruc- } \\
\text { tura industrial y } \\
\text { comercio exterior }\end{array}$ & Panel & $\begin{array}{l}\text { Hay evidencia } \\
\text { de CKA pero no } \\
\text { se calcula punto } \\
\text { de inflexión }\end{array}$ \\
\hline $\begin{array}{l}\text { Apergis } \\
(2016)\end{array}$ & $\begin{array}{l}15 \text { países del } \\
\text { mundo } \\
(1960-2013)\end{array}$ & $\begin{array}{l}\text { Emisiones de } \\
\mathrm{CO}_{2}\end{array}$ & РІB per cápita & $\begin{array}{l}\text { Panel, series } \\
\text { de tiempo y } \\
\text { cointegración }\end{array}$ & $\begin{array}{l}\text { Hay evidencia } \\
\text { de cKA sólo en } \\
12 \text { países }\end{array}$ \\
\hline $\begin{array}{l}\text { Shahbaz } \\
\text { et al (2016) }\end{array}$ & $\begin{array}{l}19 \text { países de } \\
\text { África (1971- } \\
2012)\end{array}$ & $\begin{array}{l}\text { Emisiones de } \\
\mathrm{CO}_{2}\end{array}$ & $\begin{array}{l}\text { PIB per cápita e } \\
\text { intensidad ener- } \\
\text { gética }\end{array}$ & Cointegración & $\begin{array}{l}\text { Hay evidencia } \\
\text { de CKA sólo en } 6 \\
\text { países }\end{array}$ \\
\hline $\begin{array}{l}\text { Özokcu y } \\
\text { Özdemir } \\
(2017)\end{array}$ & $\begin{array}{l}26 \text { países de la } \\
\text { ocDE y } 52 \text { paí- } \\
\text { ses emergentes } \\
(1980-2010)\end{array}$ & $\begin{array}{l}\text { Emisiones de } \\
\mathrm{CO}_{2}\end{array}$ & $\begin{array}{l}\text { РІВ per cápita y } \\
\text { uso de energía } \\
\text { per cápita }\end{array}$ & Panel & $\begin{array}{l}\text { No hay eviden- } \\
\text { cia de CKA en } \\
\text { ningún modelo } \\
\text { estimado }\end{array}$ \\
\hline
\end{tabular}

$\mathrm{SO}_{2}$ : bióxido de azufre. CO: monóxido de carbono. NOx: óxido de nitrógeno. SPM: particulas suspendidas. $\mathrm{O}_{3}$ : ozono. BOD: demanda bioquímica de oxígeno. voc: compuestos volátiles orgánicos. GEI: gases efecto invernadero. $\mathrm{N}_{2} \mathrm{O}$ : óxido nitroso. $\mathrm{CH}_{4}$ : metano. MCo: Mínimos Cuadrádos Ordinarios. Mce: Modelos de Corrección de Error. Los valores de los puntos de inflexión están estimados en dólares constantes a precios de 2005 con fines comparativos.

Fuente: elaboración propia.

Por lo tanto, la tabla 3 muestra investigaciones que analizan los efectos del ingreso sobre las emisiones y/o concentraciones de los principales GEI, destacando el $\mathrm{CO}_{2}$, el bióxido de azufre $\left(\mathrm{SO}_{2}\right)$ y el óxido de nitrógeno (NOx), así como otros impactos ambientales, entre ellos la deforestación y la contaminación de aire en diversos niveles geográficos o grupos de países mediante diferentes metodologías econométricas. Además, las estimaciones realizadas para las emisiones de $\mathrm{CO}_{2}$ per cápita (Galeotti, Lanza, \& Pauli, 2006; Itkonen, 2010; Cho, Chu \& Yang, 2014; Farhani, Mrizak, Chaibi \& Rault, 2014; Yang, Sun, Wang, \& Li, 2015; Yin, Zheng \& Cheng, 2015; Apergis, 2016; Shahbaz, Solarin \& Ozturk, 2016) indican presencia de la CKA en la mayoría de los países analizados. No obstante, también se destaca que en los estudios de Özocku 
y Özdemir (2017) y de Zilio y Caraballo (2014) -este último realizado para la región de ALC- no presentan evidencia de la CKA.

\section{La curva de Kuznets ambiental en América Latina y el Caribe: Un modelo de cointegración con panel: 1980-2015}

La evidencia presentada en la tabla 3 propone que la CKA puede ser estimada para las emisiones de GEI y que estarán básicamente en función del nivel de ingreso per cápita y de su término cuadrático. Además, en la especificación se pueden agregar variables exógenas determinantes para las trayectorias de las emisiones, entre las que se destaca el uso de combustibles fósiles (González, 2009; Hodgson, 2010). Así mismo, el uso de energías renovables ha tenido una importante participación en las matrices energéticas de algunos de los países latinoamericanos, destacando su uso en la generación de electricidad, y se puede relacionar con la eficiencia técnica y la actividad económica, puesto que permite el mejoramiento de la calidad de vida de las personas. La utilización de energía renovable es considerada una opción de sustitución de los combustibles fósiles y un insumo que ayuda en la reducción de emisiones de GEI (Sadorsky, 2009). Existen otras variables macroeconómicas que tienen influencia directa sobre la determinación de las emisiones de GEI de las cuales destacan los stocks de capital en los países altamente industrializados y en vía de desarrollo, la fuerza de trabajo, los precios de los energéticos y las variables de comercio exterior ( $\mathrm{Xu}, 2012$; Apergis \& Payne, 2009; Stern \& Common, 2001; Lee, 2005; Lee \& Lee, 2009; Suri \& Chapman, 1998).

De igual manera, es importante la relación que hay entre el comercio internacional y su contribución con emisiones de GEI en el cambio climático. Este vinculo está basado en que la liberalización comercial presenta tres efectos (омc \& PNUMA, 2009): a) el efecto escala, cuando se refiere al incremento e impactos generados por los GEI mediante la actividad económica, donde el uso de energéticos fósiles tiene una participación muy relevante; b) el efecto composición, que puede generar un efecto positivo o negativo debido a que la liberación comercial desvía la producción de un país hacia los productos que tienen ventaja comparativa, obteniendo eficiencia económica; esto se lleva a cabo siempre y cuando los sectores que tengan esa ventaja consuman menos energía en su desempeño productivo, y c) el efecto en la técnica, que busca formas más eficientes de producir y transportar bienes y servicios para contribuir a una mayor eficiencia energética, que genere menores emisiones de GEI. Esto significa que, por una parte, la liberación del comercio aumentará la disponibilidad y reducirá los costos de los productos, servicios y tecnologías que no dañen el medio ambiente, $\mathrm{y}$, por otro lado, el aumento de los ingresos 
generados del comercio puede motivar a la sociedad a demandar una mayor calidad ambiental que busque reducir las emisiones de GEI. Los impactos del comercio en el cambio climático no pueden ser determinados con anticipación, porque dependen de su magnitud e intensidad y de las estructuras económicas de los países. En consecuencia, el efecto de la apertura comercial sobre las emisiones es indeterminado, es decir, si el efecto es positivo se estaría cumpliendo con la Hipótesis de Refugio de Contaminantes (Haven Pollution Hypothesis), donde las industrias más contaminantes se relocalizan en países en los que las regulaciones ambientales son muy débiles o nulas, con el fin de evitar las legislaciones ambientales vigentes de los países desarrollados (Cole, 2004); pero si el efecto es negativo, el comercio internacional tiene efectos que benefician la calidad ambiental (Zilio \& Caraballo, 2014). Considerando lo anterior, la especificación general del modelo econométrico es la siguiente:

$$
C O_{2} p c_{i t}=\beta_{0}+\beta_{1}\left(Y p c_{i t}\right)-\beta_{2}\left(Y p c_{i t}^{2}\right)+\beta_{3}\left(E p c_{i t}\right)-\beta_{4}\left(E R p c_{i t}\right) \pm \beta_{5}\left(A C_{i t}\right)+\varepsilon_{i t}
$$

Donde $\mathrm{CO}_{2} p c_{i t}$ son las emisiones per cápita de $\mathrm{CO}_{2}$ (en toneladas por habitante); $Y p c_{i t}$ es el ingreso per cápita (en dólares por habitante a precios constantes de 2010); $Y p c^{2}{ }_{i t}$ representa al ingreso per cápita al cuadrado; $E p c_{i t}$ corresponde al consumo de energía per cápita, sin energía renovable (en barriles equivalentes de petróleo por habitante); $E R p c_{i t}$ es el consumo de energía renovable per cápita (en barriles equivalentes de petróleo por habitante) y $A C_{i t}$ simboliza el índice de apertura comercial de cada país (en porcentaje).

Para la estimación del modelo econométrico de tipo panel cointegrado se utilizó la información estadística disponible hasta el momento abarcando el período comprendido entre 1980 y 2015, y considerando únicamente 23 países de ALc: ${ }^{1}$ Argentina, Belice, Bolivia, Brasil, Chile, Colombia, Costa Rica, Cuba, Ecuador, El Salvador, Guatemala, Haití, Honduras, Jamaica, México, Nicaragua, Panamá, Paraguay, Perú, República Dominicana, Trinidad y Tobago, Uruguay y Venezuela. La muestra total está conformada por 828 observaciones.

1 Fueron seleccionaron únicamente esos 23 países de la región debido a que el resto no cuenta con información suficiente. La base de datos fue construida con información de la Comisión Económica para América Latina y el Caribe (CEPAL) en http://estadisticas. cepal.org/cepalstat/ y del Sistema de Información Económica Energética (SIEE) de la Organización Latinoamericana de Energía (OLADE) en http:/ / sier.olade.org/ 


\subsection{Metodología econométrica}

La estimación de los modelos de datos panel con cointegración estará basada en la siguiente metodología econométrica:

\subsubsection{Pruebas de heterogeneidad dinámica}

La heterogeneidad dinámica, por ejemplo la variación del intercepto entre los países y el tiempo, se puede analizar, en primer lugar, mediante una ecuación tipo Dickey-Fuller Aumentada (ADF) (Dickey \& Fuller, 1979) para cada relación en el panel, donde la hipótesis nula señala que los parámetros de la regresión son iguales en las ecuaciones utilizando como indicador una prueba $F$. En segundo lugar, se calcula una prueba tipo Chow con una distribución $F$, además de estimar un modelo autorregresivo de orden $n$ para cada una de las relaciones y probar la igualdad entre parámetros. En tercer lugar, se calcula una prueba White para grupos que tienen heteroscedasticidad, buscando comprobar la hipótesis nula de homogeneidad en la varianza de los errores a través de los países (Holz-Eakin, Newey \& Rosen, 1985; HolzEakin, 1986).

\subsubsection{Pruebas de raíces unitarias para datos panel}

Al aplicar pruebas de raíces unitarias a los datos de panel cuando son heterogéneos y no estacionarios se puede evitar problemas de regresión espuria (Constantini \& Martini, 2010). También, este tipo de pruebas se puede clasificar en dos generaciones: las que asumen independencia y las de dependencia en sección cruzada (Baltagi, 2013). En consecuencia, se estimarán tres pruebas de raíces unitarias de primera generación: 1) la LLC, que está basada en el modelo ADF, el cual restringe los parámetros $\gamma_{i}$ que son idénticos a todas las unidades transversales, permitiendo que el número de rezagos para los términos en primeras diferencias varíen también de acuerdo con las unidades transversales (Levine, Lin y \& Chu, 2002); 2) la IPS, que relaja relajan el supuesto de coeficientes autorregresivos de primer orden idénticos y permite a $\gamma$ variar en todas las unidades bajo la hipótesis alternativa (Im, Pesaran \& Shin, 2003), y 3) la H-LM, que se basa en el multiplicador de Lagrange (LM) y donde la hipótesis nula debería invertirse para cambiar a una hipótesis estacionaria con el objetivo de contar con una prueba más robusta (Hadri, 2000). 


\subsubsection{Pruebas de cointegración para panel heterogéneo}

Las técnicas de cointegración de datos panel están diseñadas para analizar información seleccionada y poder encontrar relaciones de largo plazo comunes de todo el conjunto de datos, que están al mismo tiempo asociados a la dinámica de corto plazo y cuyos efectos fijos son heterogéneos entre los diferentes miembros del panel (Pedroni, 1999). Considerando una ecuación de tipo panel:

$$
Y_{i t}=\alpha_{i t}+\delta_{i t} t+\beta_{i t} X_{i t}+e_{i t}
$$

Donde $y_{i t}$ y $X_{i t}$ son variables observables con dimensiones $\left(N^{*} T\right) \times 1$ y $\left(N^{*} T\right)$ $x m$, respectivamente, puede haber presencia de heterogeneidad tanto en la dinámica como en la varianza de los errores en los datos panel. Por lo tanto, Pedroni $(1999 ; 2004)$ propone dos conjuntos de pruebas para cointegración, las cuales plantean la hipótesis nula de no cointegración, $\rho_{i}=1$ tomando en cuenta el siguiente proceso autorregresivo de los residuales:

$$
e_{i t}=\rho_{i} e_{i t-1} u_{i t}
$$

Las primeras pruebas de cointegración están basadas sobre el enfoque within dimension (dentro de la dimensión) con estadísticos de panel cointegrado determinados como (ecuaciones 6 a la 9): panel $v$, panel $\rho$, panel PP (Phillips \& Perron, 1988) y panel ADF y donde estos estadísticos juntan los coeficientes autorregresivos a través de las diferentes unidades o individuos para las pruebas de raíces unitarias sobre los residuales estimados y toman en cuenta la heterogeneidad y los factores de tendencia común entre las unidades (Pedroni, 1999; Apergis \& Payne, 2009; Lee, 2005):

Panel v-estadístico: $Z_{v}=\left(\sum_{i=1}^{N} \sum_{t=1}^{T} \hat{L}_{11 i}^{-2} \hat{e}_{i t-1}^{2}\right)^{-1}$

Panel $\rho$-estadístico: $Z_{\rho}=\left(\sum_{i=1}^{N} \sum_{t=1}^{T} \hat{L}_{11 i}^{-2} \hat{e}_{i t-1}^{2}\right)^{-1} \sum_{i=1}^{N} \sum_{t=1}^{T} \hat{L}_{11 i}^{-2}\left(\hat{e}_{i t-1}^{2} \hat{e}_{i t}-\hat{\lambda}_{i}\right)$

Panel pP-estadístico: $Z_{t}=\left(\hat{\sigma}^{2} \sum_{i=1}^{N} \sum_{t=1}^{T} \hat{L}_{11 i}^{-2} \hat{e}_{i t-1}^{2}\right)^{-1 / 2} \sum_{i=1}^{N} \sum_{t=1}^{T} \hat{L}_{11 i}^{-2}\left(\hat{e}_{i t-1} \hat{e}_{i t}-\hat{\lambda}_{i}\right)$

Panel ADF-estadístico: $Z_{t}^{*}=\left(\hat{S}^{2} \sum_{i=1}^{N} \sum_{t=1}^{T} \hat{L}_{11 i}^{-2} \hat{e}_{i t-1}^{2}\right)^{-1 / 2} \sum_{i=1}^{N} \sum_{t=1}^{T} \hat{L}_{11 i}^{-2} \hat{e}_{i t-1}^{*} \Delta \hat{e}_{i t}^{*}$ 
Además, están las pruebas que se basan en el enfoque between dimension (entre dimensión) (estadísticos de cointegración de panel de grupos promedio) que se componen de tres estadísticos (ecuaciones 10, 11 y 12): grupo $\rho$, grupo PP y grupo ADF y están establecidos sobre promedios de coeficientes autorregresivos individuales asociados con las pruebas de raíces unitarias de los residuales de cada una de las unidades (Pedroni, 1999; Apergis \& Payne, 2009; Lee, 2005):

Grupo $\rho$-estadístico: $\tilde{Z}_{\rho}=\sum_{i=1}^{N}\left(\sum_{t=1}^{T} \hat{e}_{i t-1}^{2}\right)^{-1} \sum_{t=1}^{T}\left(\hat{e}_{i t-1}^{*} \Delta \hat{e}_{i t}^{*}-\hat{\lambda}_{i}\right)$

Grupo PP-estadístico $\tilde{Z}_{t}=\sum_{i=1}^{N}\left(\hat{\sigma}^{2} \sum_{t=1}^{T} \hat{e}_{i t-1}^{2}\right)^{-1 / 2} \sum_{t=1}^{T}\left(\hat{e}_{i t-1}^{*} \Delta \hat{e}_{i t}^{*}-\hat{\lambda}_{i}\right)$

Grupo ADF-estadístico: $\tilde{Z}_{t}^{*}=\sum_{i=1}^{N}\left(\sum_{t=1}^{T} \hat{s}_{i}^{2} \hat{e}_{i t-1}^{* 2}\right)^{-1 / 2} \sum_{t=1}^{T}\left(\hat{e}_{i t-1}^{*} \Delta \hat{e}_{i t}^{*}\right)$

Donde, $\hat{e}_{i t}$ son los residuales estimados de la ecuación 4 y $\hat{L}_{11 i}^{2}$ es la matriz de covarianza estimada de largo plazo para $\Delta \hat{e}_{i t}$. Por su parte, $\hat{\sigma}_{i}^{2}$ y $\hat{s}_{i}^{2}\left(\hat{s}_{i}^{* 2}\right)$ son, respectivamente, las varianzas de largo plazo y actuales de las $i$ unidades o individuos. Los estadísticos están distribuidos asintóticamente de forma normal. El estadístico panel $v$ es una prueba unilateral donde valores altos positivos rechazan la hipótesis nula de no cointegración. El resto de los estadísticos divergen hacia valores negativos y, por lo tanto, si son más negativos se rechazará la hipótesis nula (Lee, 2005).

La prueba de cointegración de Kao (1999) considera que las variables son no estacionarias de orden de integración I(1) y propone pruebas tipo DickeyFuller (DF) y ADF, donde la hipótesis nula es la existencia de no cointegración $\left(H_{\mathrm{o}}: \rho=1\right)$. La prueba tipo DF está calculada mediante efectos fijos en los residuales. Considerando la ecuación 13:

$$
\hat{e}_{i t}=\rho \hat{e}_{i t-1}+\sum_{j=1}^{p} \vartheta_{j} \Delta \hat{e}_{i t}+v_{i t p}
$$

Se puede obtener la prueba ADF (Baltagi, 2013; Kao, 1999):

$$
A D F=\frac{t_{A D F}+\frac{\sqrt{6 N \partial_{v}}}{2 \partial_{0 v}}}{\sqrt{\frac{\partial_{0 v}^{2}}{2 \partial_{v}^{2}}+\frac{3 \partial_{v}^{2}}{10 \partial_{0 v}^{2}}}}
$$


Donde, $t_{A D F}$ es el estadístico $t$ de $\rho$ en la ecuación 13, además que $\hat{\sigma}_{v}^{2}=\hat{\Sigma}_{y y}-\hat{\Sigma}_{y x} \hat{\Sigma}_{x x}^{-1}$ y $\hat{\sigma}_{0 v}^{2}=\hat{\Omega}_{y y} \hat{\Omega}_{y x} \hat{\Omega}_{x x}^{-1}$. La prueba ADF converge a una distribución normal $\mathrm{N}(0,1)$.

3.1.4. Relaciones de largo plazo mediante Fully Modified Ordinary Least Squares (FMOLS)

El método de Fully Modified Ordinary Least Squares (FMOLs) (Mínimos Cuadrados Ordinarios Modificados Completamente) es un enfoque semiparamétrico desarrollado por Phillips y Hansen (1990), Phillips y Moon (1999) y Pedroni (2000) para la estimación de una sola relación de cointegración, mediante la combinación de variables no estacionarias con orden de integración I(1) y realiza correcciones apropiadas para eliminar los problemas de inferencia con el método estático de Engle y Granger (1987), por lo que las pruebas $t$ de los coeficientes estimados de largo plazo son válidos, debido a que se realiza una ponderación en dichos coeficientes. El método usa estimadores Kernel de los parámetros de perturbación que afectan la distribución asintótica de los estimadores obtenidos por Mco para dar cuenta de los efectos de correlación de las series y las pruebas de endogeneidad de los regresores resultado de la existencia de relaciones de cointegración (Shahbaz, 2009). Entonces, una estimación de Mco típica de datos panel busca encontrar un estimador $\beta$ mediante FMoLs que represente el vector de cointegración de la relación de largo plazo entre el conjunto de variables $y_{i t} \mathrm{y} X_{i t}$ (Pedroni, 2006; Kao \& Chiang, 2000):

$$
\hat{\beta}_{F M}=\left[\Sigma_{i=1}^{N} \Sigma_{t=1}^{T}\left(X_{i t}-\bar{X}_{i}\right)\left(X_{i t}-\bar{X}_{i}\right)^{\prime}\right]^{-1}\left[\Sigma_{i=1}^{N}\left(\Sigma_{t=1}^{T}\left(X_{i t}-\bar{X}_{i}\right) \hat{y}_{i t}^{+}-T \Delta_{\varepsilon v}^{+}\right)\right]
$$

Donde $\Delta_{\varepsilon v}^{+}$es el término de corrección de correlación serial y $\hat{y}_{i t}^{+}$es el término de corrección de endogeneidad.

\subsubsection{Pruebas de causalidad de Granger}

La prueba de causalidad de Granger (Granger, 1997; Engle \& Granger, 1987; Sims, 1972; Pindyck \& Rubinfeld, 2001) emplea un conjunto de variables que sean estacionarias, debido a que, si se utilizarán series no estacionarias, la prueba de causalidad podría conducir a resultados de causalidad espurios (Granger \& Newbold, 1974). Por lo tanto, se usa el método de Engle y Granger (1987) de dos etapas: en la primera etapa, se lleva a cabo la estimación 
del modelo de largo plazo de la ecuación, y en la segunda etapa se obtiene la serie de los residuales que busca incorporar información de las propiedades de cointegración de las series, las cuales especifican la relación causal entre dos o más variables, conteniendo una relación de equilibrio e incluyendo el vector de cointegración que es representado como mecanismo de corrección de error $\left(\varepsilon_{i t-1}\right)$, dando evidencia de la existencia de una relación de largo plazo y que persistirá más allá del ajuste de corto plazo (Johnston \& Dinardo, 2001).

$$
\Delta y_{i t}=\alpha+\sum_{j=1}^{K} \gamma_{i t} \Delta X_{i t-n}+\sum_{j=1}^{K} \delta_{i t} \Delta y_{i t-n}+\varepsilon_{i t-1}+v_{i t}
$$

\subsection{Evidencia de la curva de Kuznets ambiental para América Latina y el Caribe: 1980-2015}

De acuerdo con la metodología planteada, se realizaron, en primer lugar, las pruebas para identificar la posible existencia de heterogeneidad dinámica del modelo de panel de la CKA para toda la región. Los resultados se muestran en la tabla 4, los cuales señalan que en la prueba $\operatorname{ADF}(2)$ se rechaza la hipótesis nula de la existencia de igualdad entre los parámetros. Así mismo, la prueba AR(2) también rechaza la hipótesis nula de la existencia de homogeneidad en los parámetros de sección cruzada al igual que en la prueba White, por lo que existe heterogeneidad en la varianza de los errores a través de los países.

Tabla 4. Pruebas de heterogeneidad dinámica sobre variables de América Latina y el Caribe

\begin{tabular}{cccc}
\hline Especificación & Prueba ADF(2) & Prueba AR(2) & Prueba White \\
\hline $\mathrm{CO}_{2} p c-Y p c-Y p c^{2}-E p c-E R p c-A P$ & F: $37.50(0.000)^{*}$ & F: $88.43(0.000)^{*}$ & $\chi^{2}: 110.09(0.000)^{*}$ \\
\hline$P$
\end{tabular}

Período: 1980-2015 (828 observaciones).

* Indica $5 \%$ del nivel de significancia. Los valores entre paréntesis indican la probabilidad de la prueba. ADF reporta igualdad en los parámetros a través de los paneles, AR muestra una relación autorregresiva de tercer orden y White indica igualdad en la varianza a través de los paneles.

Fuente: elaboración propia.

Las pruebas de raíces unitarias de panel (tabla 5) indican que en el caso de LLC e IPS, en cada una de las series en niveles con y sin efectos temporales se acepta la hipótesis nula de existencia de raíces unitarias en las series de panel, tanto a nivel común en el grupo como individual. Aplicando la primera diferencia a las series sin efectos temporales los resultados muestran que son estacionarias, por lo que su orden de integración es I(1). En la prueba LLC, al 
aplicar los efectos temporales en primeras diferencias, se acepta la hipótesis de existencia de raíz unitaria común en las series, debido a que la tendencia determinística la convierte en una serie no estacionaria o en una caminata aleatoria. En la prueba H-LM, las series en niveles rechazan la hipótesis nula de la existencia en general de estacionariedad, a diferencia de las mismas series en las primeras diferencias, donde se concluye que son estacionarias tanto en los casos con y sin efectos temporales. Además, se comprueba la existencia de raíces unitarias en el PIB o ingreso per cápita, siendo una señal de la presencia de efectos permanentes como consecuencia de factores como los shocks tecnológicos, las fluctuaciones económicas o los impactos en las demandas agregadas, que tienen efectos sobre los niveles de producción (Lee \& Lee, 2009; Narayan, 2004).

Tabla 5. Raíces unitarias para datos panel sobre variables de América Latina y el Caribe

\begin{tabular}{|c|c|c|c|c|c|c|}
\hline \multirow{2}{*}{ Variable } & \multicolumn{2}{|c|}{$\begin{array}{l}\text { Levine, Lin y Chu (2002) } \\
\text { (LLC) }\end{array}$} & \multicolumn{2}{|c|}{$\begin{array}{c}\text { Im, Pesaran y Shin (1997) } \\
\text { (IPS) }\end{array}$} & \multicolumn{2}{|c|}{$\begin{array}{l}\text { Hadri (2000) } \\
\quad(\mathrm{H}-\mathrm{LM})\end{array}$} \\
\hline & $\begin{array}{l}\text { Sin efectos } \\
\text { temporales }\end{array}$ & $\begin{array}{l}\text { Con efectos } \\
\text { fijos tempo- } \\
\text { rales }\end{array}$ & $\begin{array}{l}\text { Sin efectos } \\
\text { temporales }\end{array}$ & $\begin{array}{c}\text { Con efectos } \\
\text { fijos tempo- } \\
\text { rales }\end{array}$ & $\begin{array}{l}\text { Sin efectos } \\
\text { temporales }\end{array}$ & $\begin{array}{c}\text { Con efectos } \\
\text { fijos tempo- } \\
\text { rales }\end{array}$ \\
\hline $\mathrm{CO}_{2} p c_{i t}$ & $\begin{array}{c}0.017 \\
(0.507)\end{array}$ & $\begin{array}{l}-1.563 \\
(0.060)\end{array}$ & $\begin{array}{c}1.732 \\
(0.958)\end{array}$ & $\begin{array}{l}-1.843 \\
(0.633)\end{array}$ & $\begin{array}{c}13.028 \\
(0.000)^{*}\end{array}$ & $\begin{array}{c}9.244 \\
(0.000)^{*}\end{array}$ \\
\hline$\Delta C \mathrm{O}_{2} p c_{i t}$ & $\begin{array}{l}-24.484 \\
(0.000)^{*}\end{array}$ & $\begin{array}{l}-23.359 \\
(0.000)^{*}\end{array}$ & $\begin{array}{l}-25.387 \\
(0.000)^{*}\end{array}$ & $\begin{array}{l}-24.316 \\
(0.000)^{*}\end{array}$ & $\begin{array}{c}0.642 \\
(0.260)\end{array}$ & $\begin{array}{c}7.867 \\
(0.000)^{*}\end{array}$ \\
\hline $\mathrm{Ypc}_{\mathrm{it}}$ & $\begin{array}{c}5.731 \\
(1.000)\end{array}$ & $\begin{array}{c}-5.781 \\
(0.000)^{*}\end{array}$ & $\begin{array}{c}8.410 \\
(0.999)\end{array}$ & $\begin{array}{l}-3.916 \\
(0.000)^{*}\end{array}$ & $\begin{array}{c}13.818 \\
(0.000)^{*}\end{array}$ & $\begin{array}{c}10.765 \\
(0.000)^{*}\end{array}$ \\
\hline$\Delta Y p c_{i t}$ & $\begin{array}{l}-13.233 \\
(0.000)^{*}\end{array}$ & $\begin{array}{l}-11.219 \\
(0.000)^{*}\end{array}$ & $\begin{array}{l}-14.104 \\
(0.000)^{*}\end{array}$ & $\begin{array}{l}-11.891 \\
(0.000)^{*}\end{array}$ & $\begin{array}{c}3.843 \\
(0.000)^{*}\end{array}$ & $\begin{array}{c}4.751 \\
(0.000)^{*}\end{array}$ \\
\hline$Y p c^{2}{ }_{i t}$ & $\begin{array}{c}6.119 \\
(1.000)\end{array}$ & $\begin{array}{c}-5.893 \\
(0.000)^{*}\end{array}$ & $\begin{array}{c}8.720 \\
(0.999)\end{array}$ & $\begin{array}{l}-3.935 \\
(0.000)^{*}\end{array}$ & $\begin{array}{c}13.940 \\
(0.000)^{*}\end{array}$ & $\begin{array}{c}10.743 \\
(0.000)^{*}\end{array}$ \\
\hline$\Delta Y p c^{2}{ }_{i t}$ & $\begin{array}{l}-13.338 \\
(0.000)^{*}\end{array}$ & $\begin{array}{l}-11.201 \\
(0.000)^{*}\end{array}$ & $\begin{array}{l}-13.991 \\
(0.000)^{*}\end{array}$ & $\begin{array}{l}-11.924 \\
(0.000)^{*}\end{array}$ & $\begin{array}{c}3.739 \\
(0.000)^{*}\end{array}$ & $\begin{array}{c}4.978 \\
(0.000)^{*}\end{array}$ \\
\hline $\mathrm{Epc}_{\mathrm{it}}$ & $\begin{array}{c}0.123 \\
(0.549)\end{array}$ & $\begin{array}{c}-0.485 \\
(0.314)\end{array}$ & $\begin{array}{c}1.205 \\
(0.886)\end{array}$ & $\begin{array}{c}-0.597 \\
(0.275)\end{array}$ & $\begin{array}{c}14.177 \\
(0.000)^{*}\end{array}$ & $\begin{array}{c}8.525 \\
(0.000)^{*}\end{array}$ \\
\hline$\Delta E p c_{i t}$ & $\begin{array}{c}-21.859 \\
(0.000)\end{array}$ & $\begin{array}{l}-18.879 \\
(0.000)^{*}\end{array}$ & $\begin{array}{l}-22.584 \\
(0.000)^{*}\end{array}$ & $\begin{array}{l}-20.843 \\
(0.000)^{*}\end{array}$ & $\begin{array}{c}1.057 \\
(0.145)\end{array}$ & $\begin{array}{c}2.917 \\
(0.002)^{*}\end{array}$ \\
\hline $\mathrm{ERpc}_{\mathrm{it}}$ & $\begin{array}{c}-1.665 \\
(0.048)^{*}\end{array}$ & $\begin{array}{l}-1.010 \\
(0.156)\end{array}$ & $\begin{array}{l}-0.801 \\
(0.211)\end{array}$ & $\begin{array}{c}1.787 \\
(0.370\end{array}$ & $\begin{array}{c}11.197 \\
(0.000)^{*}\end{array}$ & $\begin{array}{c}10.927 \\
(0.000)^{*}\end{array}$ \\
\hline$\Delta E R p c_{i t}$ & $\begin{array}{l}-22.829 \\
(0.000)^{*}\end{array}$ & $\begin{array}{l}-19.463 \\
(0.000)^{*}\end{array}$ & $\begin{array}{l}-24.969 \\
(0.000)^{*}\end{array}$ & $\begin{array}{l}-23.293 \\
(0.000)^{*}\end{array}$ & $\begin{array}{c}0.861 \\
(0.195)\end{array}$ & $\begin{array}{c}3.675 \\
(0.000)^{*}\end{array}$ \\
\hline
\end{tabular}




\begin{tabular}{ccccccc}
\hline & \multicolumn{2}{c}{$\begin{array}{c}\text { Levine, Lin y Chu (2002) } \\
\text { (LLC) }\end{array}$} & \multicolumn{2}{c}{$\begin{array}{c}\text { Im, Pesaran y Shin (1997) } \\
\text { (IPS) }\end{array}$} & \multicolumn{2}{c}{$\begin{array}{c}\text { Hadri (2000) } \\
\text { Variable }\end{array}$} \\
\cline { 2 - 7 } & $\begin{array}{c}\text { Sin efectos } \\
\text { temporales }\end{array}$ & $\begin{array}{c}\text { Con efectos } \\
\text { fijos tempo- } \\
\text { rales }\end{array}$ & $\begin{array}{c}\text { Sin efectos } \\
\text { temporales }\end{array}$ & $\begin{array}{c}\text { Con efectos } \\
\text { fijos tempo- } \\
\text { rales }\end{array}$ & $\begin{array}{c}\text { Sin efectos } \\
\text { temporales }\end{array}$ & $\begin{array}{c}\text { Con efectos } \\
\text { fijos tempo- } \\
\text { rales }\end{array}$ \\
\hline \multirow{2}{*}{$\mathrm{AC}_{\text {it }}$} & -6.501 & -6.230 & -6.926 & -6.279 & 6.381 & 5.500 \\
& $(0.000)^{*}$ & $(0.000)^{*}$ & $(0.000)^{*}$ & $(0.000)$ & $(0.000)^{*}$ & $(0.000)^{*}$ \\
$\Delta A C_{i t}$ & -27.981 & -22.473 & -27.909 & -23.859 & 0.286 & 5.170 \\
& $(0.000)^{*}$ & $(0.000)^{*}$ & $(0.000)^{*}$ & $(0.000)^{*}$ & $(0.387)$ & $(0.000)^{*}$ \\
\hline
\end{tabular}

Período: 1980-2015 (828 observaciones).

Nota: para la prueba LLC: $H_{\mathrm{o}}$ : existencia de raíz unitaria los paneles vs. $H_{a}$ : el panel es estacionario. Para IPS: $H_{\mathrm{o}}$ : todos los paneles tienen raíz unitaria vs. $H_{a}$ : algunos paneles son estacionarios, y para la prueba H-LM: $H_{\mathrm{o}}$ : todos los paneles son estacionarios vs. $H_{a}$ : algunos paneles tienen raíces unitarias.

* Indica rechazo de la hipótesis nula al $5 \%$ de nivel de significancia. Los valores entre paréntesis representan la probabilidad de la prueba. Las pruebas de raíces unitarias fueron especificadas con intercepto y tendencia, y el número de rezagos fue determinado por el criterio de información de Schwarz. ( $\Delta$ ) indica que las variables están en primeras diferencias. Los valores de las variables están en logaritmos naturales.

Fuente: elaboración propia.

De acuerdo con el orden de integración de cada una de las series estadísticas, se realiza la prueba de cointegración de Pedroni $(1999 ; 2004)$ y que está dividida en dos grupos: (1) within dimension (dentro de la dimensión) y (2) between dimension (entre la dimensión), así como la prueba de cointegración de Kao (1999). Los resultados obtenidos se muestran en la siguiente tabla:

Tabla 6. Pruebas de cointegración para panel heterogéneo del modelo econométrico de la curva de Kuznets ambiental para América Latina y el Caribe

\begin{tabular}{|c|c|c|c|c|c|c|}
\hline \multicolumn{7}{|c|}{ Pedroni (1999; y 2004) } \\
\hline \multicolumn{7}{|c|}{ Within dimension (Hipótesis alternativa: Coeficientes AR comunes) } \\
\hline \multirow{2}{*}{ Estadístico } & \multicolumn{2}{|c|}{ Sin intercepto ni tendencia } & \multicolumn{2}{|c|}{ Intercepto individual } & \multicolumn{2}{|c|}{$\begin{array}{l}\text { Intercepto y tendencia } \\
\text { individual }\end{array}$} \\
\hline & Estadístico & $\begin{array}{l}\text { Estadístico } \\
\text { ponderado }\end{array}$ & Estadístico & $\begin{array}{l}\text { Estadístico } \\
\text { ponderado }\end{array}$ & Estadístico & $\begin{array}{l}\text { Estadístico } \\
\text { ponderado }\end{array}$ \\
\hline $\begin{array}{l}\text { Panel v-es- } \\
\text { tadístico }\end{array}$ & $\begin{array}{c}0.987 \\
(0.1619)\end{array}$ & $\begin{array}{l}-1.466 \\
(0.9287)\end{array}$ & $\begin{array}{c}0.552 \\
(0.291)\end{array}$ & $\begin{array}{c}-2.127 \\
(0.983)^{*}\end{array}$ & $\begin{array}{l}-0.997 \\
(0.841)\end{array}$ & $\begin{array}{l}-3.827 \\
(0.999)\end{array}$ \\
\hline $\begin{array}{l}\text { Panel } \rho-e s- \\
\text { tadístico }\end{array}$ & $\begin{array}{c}-1.789 \\
(0.037)^{*}\end{array}$ & $\begin{array}{c}-1.423 \\
(0.0774)\end{array}$ & $\begin{array}{l}-0.970 \\
(0.167)\end{array}$ & $\begin{array}{l}-0.842 \\
(0.199)\end{array}$ & $\begin{array}{c}0.565 \\
(0.714)\end{array}$ & $\begin{array}{c}0.686 \\
(0.754)\end{array}$ \\
\hline $\begin{array}{l}\text { Panel PP- } \\
\text { estadístico }\end{array}$ & $\begin{array}{l}-6.854 \\
(0.000)^{*}\end{array}$ & $\begin{array}{l}-6.752 \\
(0.000)^{*}\end{array}$ & $\begin{array}{l}-7.446 \\
(0.000)^{*}\end{array}$ & $\begin{array}{l}-8.067 \\
(0.000)^{*}\end{array}$ & $\begin{array}{l}-8.213 \\
(0.000)^{*}\end{array}$ & $\begin{array}{l}-9.555 \\
(0.000)^{*}\end{array}$ \\
\hline $\begin{array}{l}\text { Panel ADF- } \\
\text { estadístico }\end{array}$ & $\begin{array}{c}-4.675 \\
(0.000)^{*}\end{array}$ & $\begin{array}{l}-6.384 \\
(0.000)^{*}\end{array}$ & $\begin{array}{l}-5.526 \\
(0.000)^{*}\end{array}$ & $\begin{array}{l}-8.267 \\
(0.000)^{*}\end{array}$ & $\begin{array}{c}-6.287 \\
(0.000)^{*}\end{array}$ & $\begin{array}{l}-10.069 \\
(0.000)^{*}\end{array}$ \\
\hline
\end{tabular}




\begin{tabular}{|c|c|c|c|}
\hline \multicolumn{4}{|c|}{ Pedroni (1999; y 2004) } \\
\hline \multicolumn{4}{|c|}{ Between dimensión (Hipótesis alternativa: Coeficientes AR individuales) } \\
\hline \multirow{2}{*}{ Estadístico } & Sin intercepto ni tendencia & Intercepto individual & $\begin{array}{l}\text { Intercepto y tendencia } \\
\text { individual }\end{array}$ \\
\hline & Estadístico & Estadístico & Estadístico \\
\hline $\begin{array}{l}\text { Grupo } \rho- \\
\text { estadístico }\end{array}$ & $\begin{array}{l}-0.218 \\
(0.414)\end{array}$ & $\begin{array}{c}0.844 \\
(0.801)\end{array}$ & $\begin{array}{c}2.091 \\
(0.982)\end{array}$ \\
\hline $\begin{array}{l}\text { Grupo PP- } \\
\text { estadístico }\end{array}$ & $\begin{array}{c}-8.517 \\
(0.000)^{*}\end{array}$ & $\begin{array}{l}-11.039 \\
(0.000)^{*}\end{array}$ & $\begin{array}{l}-12.548 \\
(0.000)^{*}\end{array}$ \\
\hline $\begin{array}{l}\text { Grupo ADF- } \\
\text { estadístico }\end{array}$ & $\begin{array}{c}-6.142 \\
(0.000)^{*}\end{array}$ & $\begin{array}{c}-7.168 \\
(0.000)^{*}\end{array}$ & $\begin{array}{c}-8.822 \\
(0.000)^{*}\end{array}$ \\
\hline \multicolumn{4}{|c|}{ Kao (1999) } \\
\hline & Intercepto individual & & \\
\hline Estadístico & Estadístico & & \\
\hline$A D F$ & $\begin{array}{c}-3.232 \\
(0.001)^{*}\end{array}$ & & \\
\hline
\end{tabular}

Período: 1980-2015 (828 observaciones).

* Indica rechazo de la hipótesis nula de no cointegración al 5\% de nivel de significancia. Los valores entre paréntesis representan la probabilidad de la prueba. El número de rezagos fue determinado por el criterio de información de Schwarz.

Fuente: elaboración propia.

Los resultados de los estadísticos within dimension -que están basados en un modelo de efectos fijos donde cada variable explicativa tiene un sólo coeficiente, pero con distinta constante-indican que el estadístico panel $v$ acepta la hipótesis nula de no cointegración. En el caso de los tres estadísticos restantes ( $\rho$, PP y ADF) coinciden en rechazar la hipótesis nula al incluir tanto interceptos como tendencias individuales; por lo tanto, hay evidencia de la relación de cointegración entre las variables. ${ }^{2}$ Los tres estadísticos del grupo between dimension -que son estimados sobre los promedios de las variables de las unidades de corte transversal y producen estimadores entre grupos o promedios para el sistema general- muestran que tanto la especificación con intercepto y la tendencia rechazan la hipótesis nula, por lo que hay una fuerte evidencia de integración entre las series estadísticas con trayectorias muy similares en el largo plazo. Esto muestra la existencia de una relación

2 Para el estadístico $v$, el valor estimado debe ser positivo y estadísticamente significativo debido a que es una prueba unilateral y para los estadísticos $\rho$, PP y ADF, los valores calculados deben ser negativos y significativos al $5 \%$ o $10 \%$ de nivel de significancia para rechazar la hipótesis nula Pedroni $(1999 ; 2004)$. 
de estado estacionario en conjunto después de tener un efecto específico por cada país. En la prueba de Kao (1999) también existe rechazo de la hipótesis nula y consecuentemente se confirma la cointegración entre las series. Así mismo, se rechaza la posibilidad de que haya existencia de regresión espuria para el modelo de panel heterogéneo (Kao, 1999; Phillips \& Moon, 1999; Balgatti, 2013).

La existencia de cointegración entre las series de datos de panel da lugar a la estimación del modelo econométrico mediante el método FMols (Pedroni, 2000; Kao \& Chiang, 2000) con el objetivo de comprobar la existencia de la CKA. El resultado de la estimación es el siguiente (tabla 7):

Tabla 7. Estimación de la curva de Kuznets ambiental para América Latina y el Caribe mediante FMols

\begin{tabular}{cc}
\hline Variables & Coeficientes (t-estadístico) \\
\hline$Y p c_{i t}$ & $1.679(2.179)^{*}$ \\
$Y p c^{2}{ }_{i t}$ & $-0.083(-1.845)^{* *}$ \\
$E p c_{i t}$ & $0.935(10.475)^{*}$ \\
$E R p c_{i t}$ & $-0.119(-2.911)^{*}$ \\
$A C_{i t}$ & $0.030(2.656)^{*}$ \\
$R^{2}$ ajustado & 0.969 \\
\hline
\end{tabular}

Período: 1980-2015 (828 observaciones).

* $\mathrm{y}$ ** indican rechazo de la hipótesis nula al 5 y el 10\% de grado de significancia, respectivamente. Los valores entre paréntesis indican el t-estadístico de cada coeficiente. $R^{2}$ : Coeficiente de determinación. Los coeficientes estimados están representados en elasticidades.

Fuente: elaboración propia.

El modelo estimado por FMOLs indica alta correlación entre las variables de acuerdo con su coeficiente de determinación $\left(R^{2}\right)$ ajustado $(0,969)$; esto indica que las emisiones de $\mathrm{CO}_{2}$ per cápita son explicadas por cada una de las variables exógenas, debido a que son estadísticamente significativas y que cuando el ingreso per cápita aumenta en un $1 \%$, las emisiones aumentarán $1.679 \%$, mientras el término cuadrático del ingreso per cápita señala una disminución de $0,083 \%$ en las emisiones per cápita. Así mismo, existen efectos directos sobre las emisiones de $\mathrm{CO}_{2}$ per cápita cuando crece el consumo de energéticos por habitante y un efecto inverso ante el impulso de los energéticos renovables que en el largo plazo se convertirá en un factor importante para la reducción de emisiones de $\mathrm{CO}_{2}$. Por otra parte, la apertura comercial obtuvo un efecto positivo sobre las emisiones de $\mathrm{CO}_{2}$ per cápita significando que en la región se acepta la presencia de la Hipótesis de Refugio 
de Contaminantes y, en consecuencia, el efecto escala tiene un impacto mayor que los efectos técnicos y de composición. A pesar de encontrar resultados contrarios con respecto a la investigación de Zilio y Caraballo (2014) se coincide en la importancia de la variable apertura comercial. Esto representa una evidencia relevante para ALC, cuyo significado es que la región sigue siendo una opción para industrias contaminantes de los países desarrollados. Esto es consecuencia de que las legislaciones ambientales aún no son demasiado exigentes y justas en la mayoría de los países de la región de ALc.

La estimación econométrica comprueba la existencia de la hipótesis de la CKA en ALC, es decir, se cumple que en una primera etapa el nivel de emisiones de $\mathrm{CO}_{2}$ per cápita aumentará conforme se incrementen las magnitudes de las variables exógenas, posteriormente se estabilizará y, al final, decrecerá como lo indica el término cuadrático del ingreso per cápita y la magnitud en el uso de energéticos renovables. Los resultados se sustentan en la existencia de relaciones de cointegración de largo plazo en la región, los cuales llevan la estimación de vectores de corrección de error para poder inferir causalidades entre las variables mediante modelos dinámicos de corrección de error y encontrar también relaciones de corto plazo (Engle \& Granger, 1987; Granger, 1969). Los resultados se presentan en la tabla 8.

Tabla 8. Pruebas de causalidad de Granger en panel para variables de América Latina y el Caribe

\begin{tabular}{cccccccc}
\hline $\begin{array}{c}\mathrm{H}_{\mathrm{o}}: \mathrm{No} \\
\text { causa } \rightarrow\end{array}$ & $\Delta \mathrm{CO}_{2} \mathrm{pc}_{\mathrm{it}}$ & $\Delta \mathrm{Ypc}_{\mathrm{it}}$ & $\Delta \mathrm{Ypc}^{2}{ }_{\mathrm{it}}$ & $\Delta \mathrm{Epc}_{\mathrm{it}}$ & $\Delta \mathrm{ERpc}_{\mathrm{it}}$ & $\Delta \mathrm{AC}_{\mathrm{it}}$ & $\varepsilon_{\mathrm{it}-1}$ \\
\hline$\Delta \mathrm{CO}_{2} p c_{i t}$ & & 0.301 & 0.379 & 1.156 & 0.357 & 1.165 & 3.028 \\
& & $(0.824)$ & $(0.768)$ & $(0.326)$ & $(0.784)$ & $(0.322)$ & $(0.029)^{*}$ \\
$\Delta Y p c_{i t}$ & 10.436 & & 1.020 & 8.399 & 3.848 & 4.375 & 2.15190 \\
& $(0.000)^{*}$ & & $(0.383)$ & $(0.000)^{*}$ & $(0.001)^{*}$ & $(0.004)^{*}$ & $(0.092)^{* *}$ \\
$\Delta \mathrm{Ypc}^{2}{ }_{i t}$ & 10.997 & 1.379 & & 8.450 & 4.193 & 4.5183 & 2.0683 \\
& $(0.000)^{*}$ & $(0.248)$ & & $(0.000)^{*}$ & $(0.006)^{*}$ & $(0.004)^{*}$ & $(0.103)^{* *}$ \\
$\Delta E p c_{i t}$ & 7.534 & 0.328 & 0.231 & & 0.459 & 0.259 & 2.081 \\
& $(0.000)^{*}$ & $(0.805)$ & $(0.875)$ & & $(0.711)$ & $(0.854)$ & $(0.101)^{* *}$ \\
$\Delta E R p c_{i t}$ & 1.074 & 0.131 & 0.142 & 2.616 & & 0.913 & 1.873 \\
& $(0.359)$ & $(0.941)$ & $(0.9348)$ & $(0.050)^{*}$ & & $(0.434)$ & $(0.133)$ \\
$\Delta A C_{i t}$ & 1.194 & 0.246 & 0.260 & 2.471 & 0.677 & & 2.847 \\
& $(0.311)$ & $(0.864)$ & $(0.854)$ & $(0.061)^{* *}$ & $(0.566)$ & & $(0.037)^{*}$ \\
\hline
\end{tabular}

Período: 1980-2015.

${ }^{*} \mathrm{y}{ }^{* *}$ indican rechazo de la hipótesis nula a un nivel de significancia del 5 y el $10 \%$, respectivamente. El valor entre paréntesis es la probabilidad de la prueba F. $(\Delta)$ Indica que las variables están en primeras diferencias. $\varepsilon_{\mathrm{it}-1}$ representa el vector de corrección de error. Los valores de las variables están en logaritmos naturales.

Fuente: elaboración propia. 
Los estadísticos de la prueba $F$ calculados para probar la no causalidad indican que las emisiones de $\mathrm{CO}_{2}$ per cápita aceptan la hipótesis nula en su relación con cada una de las variables explicativas. En el caso del ingreso per cápita indica correspondencias de largo y corto plazo, donde hay rechazo de la hipótesis nula con respecto a las otras variables exógenas, resultando una fuerte causalidad entre ellas. El consumo de energéticos per cápita comprueba su causalidad sobre las emisiones de $\mathrm{CO}_{2}$ y la falta de causalidad entre el consumo de energías renovables y las emisiones. La variable apertura comercial sólo causa aumento en el uso de energéticos de los países.

De acuerdo con los términos monetarios, el punto de inflexión que representa el cambio en el comportamiento de las emisiones de $\mathrm{CO}_{2}$ per cápita mediante el desacoplamiento y la descarbonización para ALC es de 10.134 dólares per cápita (a precios constantes de 2010) anuales, indicando que, en 2017, esta magnitud se encuentra aún por encima del ingreso per cápita de la región considerando únicamente los 23 países en 2017 que es de 7.111 dólares (a precios constantes de 2010).

En la figura 4 se presentan las diferencias entre los ingresos per cápita actuales con respecto al punto de inflexión de la región, el cual se considerará como un indicador promedio, donde la mayoría de los países aún no ha alcanzado un nivel superior a los 10.000 dólares que contribuya, junto con el comportamiento futuro de otras variables, a sentar las bases para el inicio de la estabilización de las emisiones de $\mathrm{CO}_{2}$ per cápita y, en consecuencia, de las emisiones totales para que en etapas posteriores comiencen a presentarse tendencias descendentes. De este conjunto de países se destacan Costa Rica y México que necesitan para superar el umbral establecido entre 342 y 188 dólares de diferencia, respectivamente. Así mismo, hay seis países que han superado el punto de inflexión como son Trinidad y Tobago, Chile y Uruguay cuya diferencia es mayor a los cuatro mil dólares.

Considerando la información de los 23 países de ALC utilizada en el modelo de cointegración para datos panel de la CKA, se calculó una proyección para conocer la trayectoria de las emisiones de $\mathrm{CO}_{2}$ per cápita para la región durante las siguientes décadas. Los supuestos de crecimiento de las variables exógenas se basan en un escenario de tipo Business as usual (вAU) considerando comportamientos de acuerdo con sus crecimientos históricos. En consecuencia, se tomó en cuenta el crecimiento promedio anual de cada variable explicativa hacia el 2060: PIB per cápita: 1,277\%, consumo de energía per cápita: 0,724\%, consumo de energía renovable per cápita: 0,515\% y apertura comercial: $0,082 \%$. 
Región / países por abajo del punto inflexión

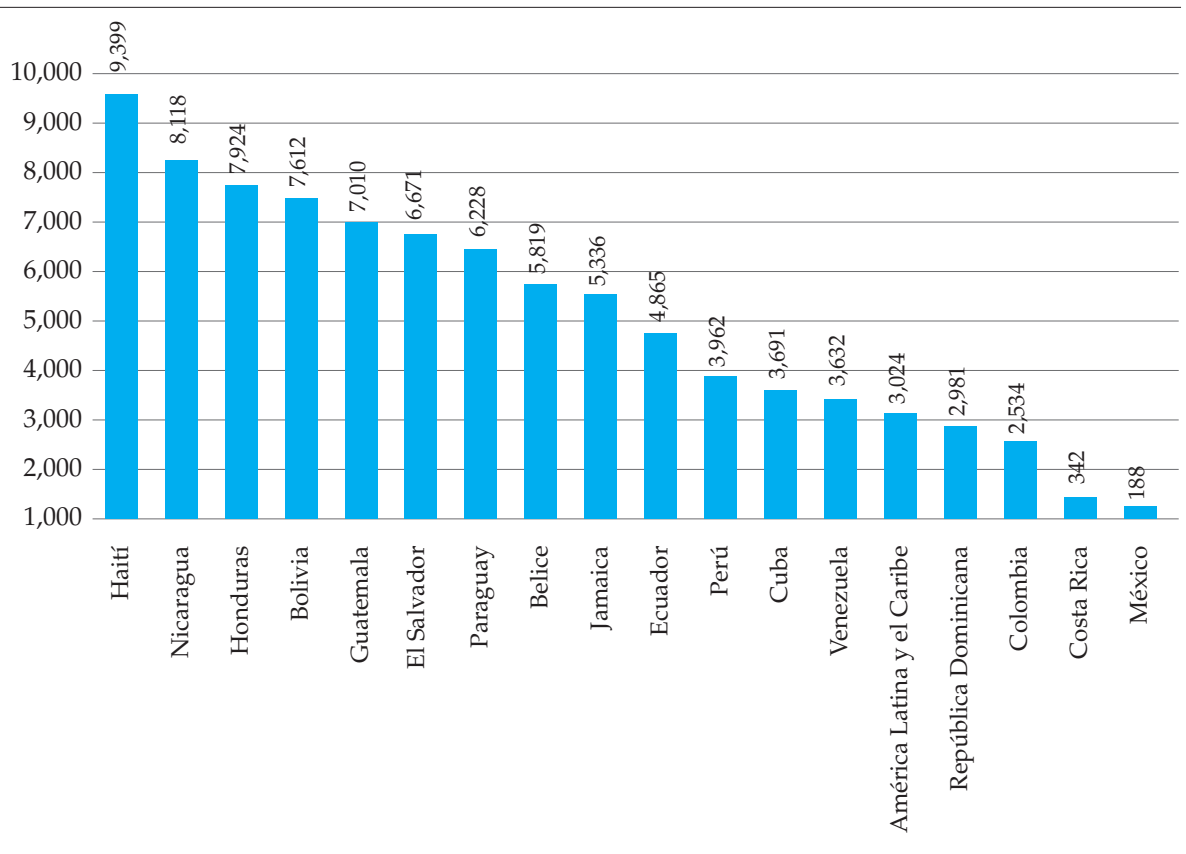

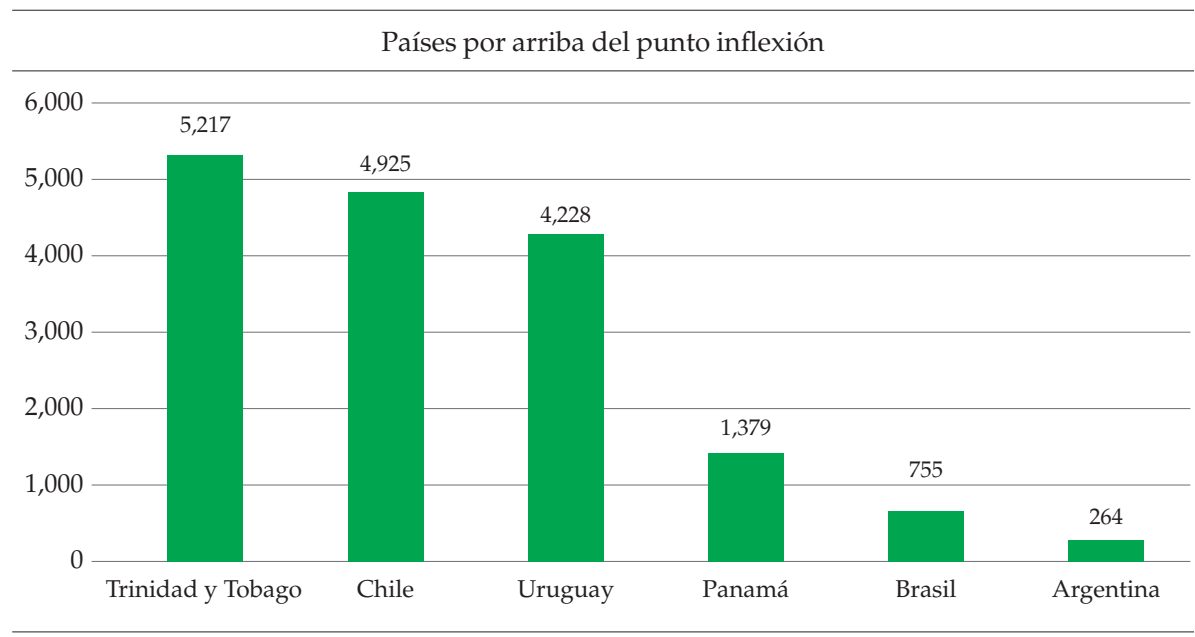

Figura 4. Diferencias monetarias entre el PIв per cápita de los países de América Latina y el Caribe en 2017 y el punto de inflexión de la curva de Kuznets ambiental estimada

(En US\$ por habitante a precios constantes de 2010)

Notas: Para América Latina y el Caribe se consideraron únicamente 23 países. Los valores del PIв per cápita de los países corresponden al año 2017, excepto Cuba y Venezuela, cuyo valor pertenece al 2016 por falta de información. Las diferencias están referidas con respecto a \$10.314 dólares por habitante obtenido de los resultados del modelo econométrico de panel estimado mediante FMOLs de la cKA para ALC.

Fuente: elaboración propia. 
En la proyección calculada de la CKA para ALc que se muestra en la figura 5 , se aprecia que la estabilización de las emisiones de $\mathrm{CO}_{2}$ per cápita de la región será entre las décadas de 2030 a 2040 de aproximadamente 3,8 toneladas por persona y, posteriormente, comenzará a disminuir la trayectoria hacia el año 2060, llegando a niveles similares a los registrados a mediados de la década de los años 2000 donde las emisiones de $\mathrm{CO}_{2}$ per cápita eran de tres toneladas por persona, aproximadamente. La comprobación de la hipótesis de la CKA para ALC en cuestión de emisiones per cápita posiblemente se deba a que, cuando se registren ingresos per cápita en la región superiores a los 10.000 dólares de acuerdo con el punto de inflexión calculado, con el impulso cada vez mayor en el uso de los energéticos renovables y con una producción y utilización más controladas de los combustibles más contaminantes mediante diversos instrumentos y políticas ambientales y energéticas.

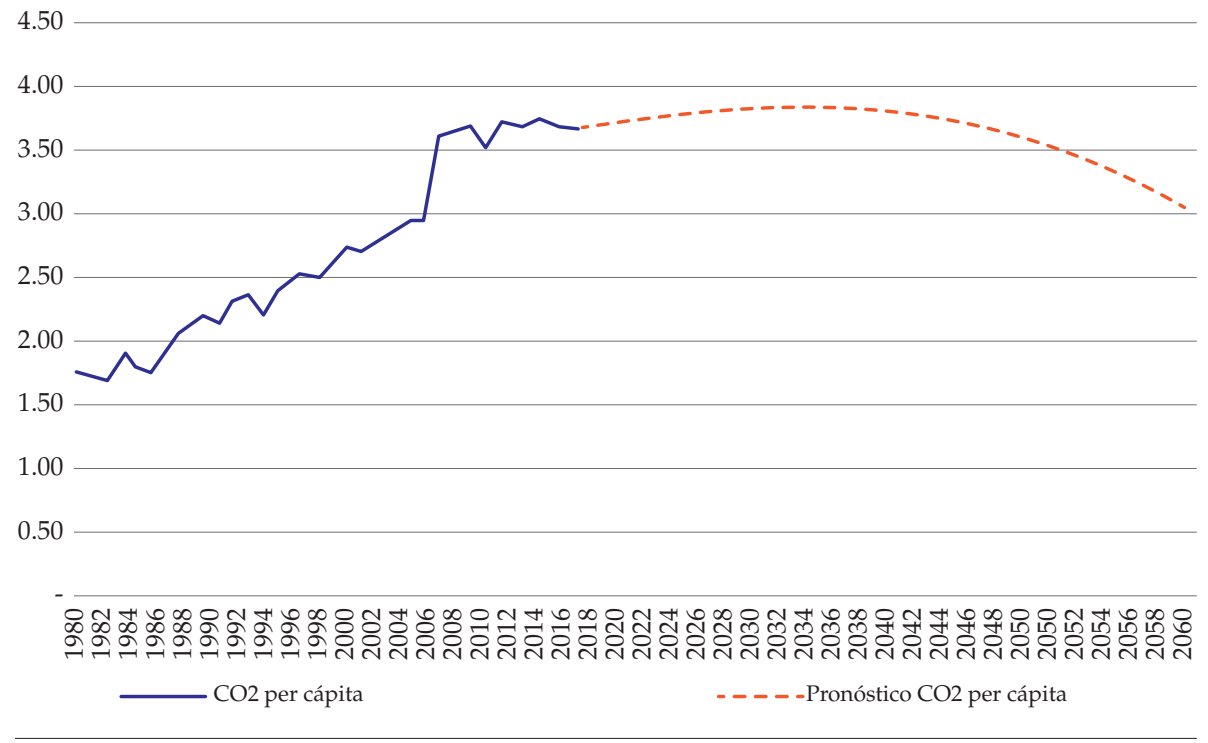

Figura 5. Trayectoria original y pronóstico al 2060 de las emisiones de $\mathrm{CO}_{2}$ per cápita mediante la curva de Kuznets ambiental en América Latina y el Caribe (en toneladas por habitante)

Periodos: Serie original: 1980-2015. Pronóstico: 2016-2060.

Notas: Se consideró únicamente la información correspondiente a los 23 países de ALc. Los supuestos de crecimiento de las variables exógenas se basan en sus crecimientos promedio anuales históricos.

Fuente: elaboración propia con base en el modelo cointegrado de panel estimado mediante FMOLs de la CKA para ALC. 


\section{Discusión de resultados e implicaciones de política}

El cambio climático ha provocado en la región de ALC diversas alteraciones climáticas e impactos que se han manifestado de forma negativa sobre los seres vivos y ejercer una presión extra sobre los recursos naturales. Los escenarios hacia finales del siglo xxI no son optimistas, debido a que la tendencia de la temperatura de la superficie global podría aumentar entre $1^{\circ} \mathrm{C}$ a $3,7^{\circ} \mathrm{C}$.

En las últimas cuatro décadas en ALC, la situación económica ha registrado un crecimiento moderado cercano al 2,54\% anual. La magnitud de este crecimiento y el comportamiento ascendente de la población latinoamericana ha generado que los niveles de PIB per cápita sean demasiado bajos, registrando para la región una tasa de crecimiento anual de 0,97\%, en promedio. Así mismo, el uso de energía, tanto total como en términos per cápita, han presentados crecimientos constantes determinados por las particulares características de los sectores energéticos y las actividades económicas de cada uno de los países que la componen. Es importante mencionar que ALc no está exenta y es sensible a los impactos de las crisis petroleras y de las fluctuaciones en los precios de los energéticos, en consecuencia, y asociado a estos indicadores macroeconómicos, las emisiones de GEI, en particular el $\mathrm{CO}_{2}$, también ha tenido aumentos significativos donde la región está contribuyendo con cerca del $10 \%$ de las emisiones mundiales.

La evidencia para ALC, estimada mediante el modelo de panel cointegrado, señala la comprobación de la existencia de la hipótesis de la CKA, contrario a la evidencia que presenta la investigación de Zilio y Caraballo (2014), y donde los países de la región deben alcanzar, en promedio, un nivel de 10.314 dólares per cápita anuales para poder estabilizar sus emisiones de $\mathrm{CO}_{2}$ y, posteriormente, comenzar a reducirlas iniciando un proceso de actividad económica baja en carbono. Sólo 6 de los 23 países analizados correspondientes a la región han superado el umbral de los 10.000 dólares. La estimación de la CKA para ALC da pie a la simulación de un escenario de las emisiones de $\mathrm{CO}_{2}$ per cápita hacia el año 2060 el cual indica que para los siguientes 20 años se podría alcanzar un periodo de estabilización de emisiones y posteriormente comenzarían a disminuir llegando a niveles cercanos de tres toneladas por habitante al final del periodo estimado. Esta trayectoria es resultado de los incrementos significativos en los ingresos per cápita por parte de los países de la región por arriba de los 10.000 dólares, y del uso de las energías renovables las cuales podrían ser un importante impulsor para que las economías tiendan hacia una senda baja en carbón.

Los indicadores y resultados presentados en este documento son una breve evidencia de que el estilo de desarrollo en los países de ALc no es sostenible, 
por lo que deben mejorar sus condiciones económicas, ambientales y sociales implementando diversas medidas de adaptación y mitigación, que ayuden a enfrentar los impactos del cambio climático -principalmente en el Caribe-, y poder contribuir con la reducción de emisiones de GEI a nivel mundial (PNUMA \& CEPAL, 2010; CEPAL, 2015; Sánchez \& Reyes; 2015). Por lo tanto, las actividades económicas de la región deberán tener entre sus objetivos el de generar un mayor dinamismo, que produzca cambios en la base productiva y permita adaptarse a diversos cambios tecnológicos en la forma de producción, además de buscar que cambien los patrones de consumo de bienes y servicios hacia unos más sostenibles (CEPAL, 2015; Bárcena, et al., 2017); esto debe estar asociado con la implementación y cumplimiento de diversas políticas públicas al interior de los países, así como el acatamiento de los acuerdos internacionales basados en el desarrollo sostenible. Además, las tendencias ascendentes del consumo de energía y su relación con las emisiones de $\mathrm{CO}_{2}$ presentadas desde 1980 hacia recientes años son preocupantes, por lo que los países de la región deben mejorar su eficiencia energética siguiendo con la tendencia de construir sistemas energéticos que generen menos emisiones de $\mathrm{CO}_{2}$, a través de la reducción de la demanda de energía y el cambio hacia energías más limpias como son los biocombustibles y la hidroenergía que ayuden al desacoplamiento entre crecimiento económico y emisiones (Bárcena et al., 2017).

Para que la región de ALc pueda impulsar su crecimiento y desarrollo económicos, modificar el sector energético y su demanda, así como disminuir sus emisiones de $\mathrm{CO}_{2}$, se sugiere implementar diversas políticas fiscales ambientales, a través de los impuestos o subsidios verdes, que pueden contribuir a reducir o, en algunos casos, eliminar las externalidades negativas sobre medio ambiente (Bárcena et al., 2017). Los recursos obtenidos de la recaudación fiscal ambiental pueden apoyar a consolidar el balance de las finanzas públicas, disminuir la carga fiscal en otros rubros para los contribuyentes y disponer de recursos fiscales adicionales, además de que pueden ser destinados a programas sociales. Del mismo modo, gravar el uso de energéticos genera una fuente considerable de ingresos al gobierno que también pueden destinarse a fines específicos ambientales, a infraestructura vial que podría ser vista como un tipo de derecho de uso, a la adquisición de nuevas tecnologías para captar y a reducir emisiones de GEI (HSU, 2011).

Debido a la complejidad del análisis económico de un fenómeno global como es el cambio climático y su vinculación con la hipótesis de la CKA, las estimaciones y resultados obtenidos para ALc no están exentos de limitaciones. En primer lugar, considerando el diseño cuantitativo, la determinación de variables macroeconómicas relevantes está basada en la literatura disponible, 
donde pueden existir otras variables que no fueron consideradas. En segundo lugar, el análisis de la CKA para ALC se enfoca en la en la demanda, siendo una buena aproximación hacia el comportamiento de los consumidores; sin embargo, es importante considerar para futuros análisis la conducta del lado de la oferta, principalmente de los propietarios de recursos como son los combustibles fósiles. Es conocido que la mayoría de las medidas de mitigación de GEI propuestas y llevadas a cabo para disminuir los impactos del cambio climático y buscar una eficiencia económico-ambiental no tienen el impacto deseado debido a que omiten las acciones que realizan los productores. Este tipo de planteamiento es expresado por Sinn (2012), que ejemplifica el caso de los propietarios de los combustibles fósiles, donde la oferta de energéticos, generalmente, es inelástica a los movimientos de sus precios, independientemente del comportamiento de la demanda. No obstante, los productores de combustibles fósiles pueden provocar una desestabilización en cuanto al objetivo mundial de disminuir las emisiones de $\mathrm{CO}_{2}$ cuando sean afectadas sus ganancias por fallas de mercado, como pueden ser la incertidumbre de los cambios en los precios de los energéticos en el futuro, la expropiación de recursos en países inestables políticamente o la aplicación de políticas sustentables que no sean benéficas para ellos. Estas causas podrían generar una extracción mayor de combustibles fósiles en el presente, que harán que aumenten las emisiones y concentraciones de $\mathrm{CO}_{2}$ en la atmósfera y, por ende, expandir la magnitud de los impactos del cambio climático en periodos de tiempo más cortos en contraste con lo estimado por la evidencia científica.

\section{Agradecimientos}

Agradecemos los comentarios y sugerencias del Mtro. Manuel García Álvarez de la Facultad de Economía de la unAm y de los dictaminadores anónimos.

\section{Descargos de responsabilidad}

Las opiniones y los errores son responsabilidad exclusiva de los autores y no representan posiciones institucionales.

\section{Referencias bibliograficas}

Al Sayed, A., \& Sek, S. (2013). Environmental Kuznets curve: Evidences from developed and developing economies. Applied Mathematical Sciences, 7(22), 1081-1092.

Alam, M.S. (2006). Economic Growth with Energy. MPRA Paper 1260. 
Anderson, D., \& Cavendish, W. (2001). Dynamic Simulation and environmental policy analysis: Beyond comparative statics and the environmental Kuznets curve. Oxford Economic Papers, 53, 721-746.

Ang, J. (2007). $\mathrm{CO}_{2}$ Emissions, energy consumption and output in France. Energy Policy, 35, 4772-4778.

Li, H., Grijalva, T., \& Berrens, R. (2007). Economic growth and environmental quality: A meta-analysis of environmental Kuznets curve studies. Economics Bulletin, 17(5), 1-11.

Apergis, N. (2016). Environmental Kuznets curves: New evidence on both panel and country-level $\mathrm{CO}_{2}$ emissions. Energy Economics, 54, 263-271.

Apergis, N. y, \& Payne, J. (2009). Energy consumption and economic growth in central america: Evidence from a panel cointegration and error correction model. Energy Economics, 31, 211-216.

Baltagi, B.H. (2013). Econometric Analysis of Panel Data. Reino Unido: John Wiley \& Sons Ltd.

Bárcena, A., Samaniego, J., Galindo, L.M., Ferrer, J., Alatorre, J.E., Stockins, P., Reyes, O., Sánchez, L., \& Mostacedo, J. (2017). La economía del cambio climático en América Latina y el Caribe. Una visión gráfica. Santiago de Chile: CEPAL.

CEPAl (2015). La economía del cambio climático en América Latina y el Caribe. Paradojas y desafios del desarrollo sostenible. Santiago de Chile: CEPAL.

CEPAL, OLADE, \& GTZ (2003). Energía y Desarrollo Sustentable en América Latina y el Caribe. Guía para la Formulación de Politicas Energéticas. Santiago de Chile: CEPAL.

Cho, C.H., Chu, Y. P., \& Yang, H. Y. (2014). An environment Kuznets curve for GHG emissions: A panel cointegration analysis. Energy Sources, Part B: Economics, Planning and Policy, 9(2), 120-129.

Cole, M. (2004). Trade, the pollution haven hypothesis and environmental Kuznets curve: Examining the linkages. Ecological Economics, 48, 71-81.

Cole, M., Rayner, A., \& Bates, J. (1997). The environmental Kuznets curve: an empirical analysis. Environment and Development Economics, 2(4), 401-416.

Common, M., \& Stagl, S. (2008). Introducción a la Economía Ecológica. Editorial Reverté: España.

Constantini, V., \& Martini, C. (2010). The causality between energy consumption and economic growth: A multi-sector analysis using non-stationary cointegrated panel data. Energy Economics, 32, 591-603.

Dickey, D.A., \& Fuller, W.A. (1979). Distribution of the estimators for autorregresive time series with a unit root. Journal of the American Statistical Association, 74, 417-431. 
Ekins P. (2000). Economic Growth and Environmental Sustainability. Londres: Routledge.

Engle, R.F., \& Granger, C.W.J. (1987). Co-integration and error correction: Representation, estimation and testing. Econometrica, 55(2), 251-276.

Fan, C., \& Zheng, X. (2013). An empirical study of the environmental Kuznets curve in sichuan province, China. Environment and Pollution, 2(3), 107-115.

Farhani, S., Mrizak, S., Chaibi, A., \& Rault, C. (2014). The environmental Kuznets curve and sustainability: A panel data analysis. Energy Policy 71, 189-198.

Ferrer, A. (1998). América Latina y la globalización. Revista CEPAL, núm. extraordinario, 155-168.

Galeotti, M., Lanza, A., \& Pauli, F. (2006). Reassessing the environmental Kuznets curve for $\mathrm{CO}_{2}$ emissions: A robustness exercise. Ecological Economics, 57, 152-163.

Goldemberg, J., Johansson, T., Reddy, A., \& Williams, R. (1987). Energy for a Sustainable World. Washington, DC: World Resources Institute.

Goldman, B. (2012). Meta-analysis of environmental Kuznets curve studies: Determining the cause of the curve's presence. Honors Projects, 117, 1-21. González, J. (2009). Energías Renovables. España: Editorial Reverté.

Granger, C. W. J., \& Newbold, P. (1974). Spurious regression in econometrics. Journal of Econometrics, 2, 111-120.

Granger, C. W. J. (1969), Investigating causal relations by econometric models and cross spectral methods. Econometrica, 37, 424-438.

Granja, F., Mendonca, A., \& Nogueira, J. (2002). Poverty and Environmental Degradation: The Kuznets Environmental Curve for the Brazilian Case. Texto para Discusión 267, Universidad de Brasilia.

Grossman, G., \& Krueger, A. (1991). Environmental Impact of a North American Free Trade Agreement. NBER Working Paper 3914.

Hadri, K. (2000). Testing for stationary in heterogeneous panel data. Econometrics Journal, 3, 148-161.

Hodgson, P.E. (2010). Energy, the Environment and Climate Change. Londres: Imperial College Press.

Holz-Eakin, D. (1986). Testing for Individual Effects in Dynamic Models Using Panel Data. NBER Technical Working Paper 57.

Holz-Eakin, D., Newey, W., \& Rosen, H. (1985). Implementing Causality Tests with Panel Data with an Example from Local Public Finance. NBER Technical Working Paper 48.

Hsu, S.L. (2011). The Case for a Carbon Tax. Estados Unidos: Island Press.

Im, K.S., Pesaran, M.H., \& Shin, Y. (2003). Testing for unit roots in heterogeneous panel. Journal of Econometrics, 115, 53-74. 
IPCC (2013). Contribution of working group i to the fifth assessment report of the intergovernmental panel on climate change, Climate Change 2013: The Physical Science Basis: Final Draft Underlying Scientific-Technical Assessment. Stockholm.

IPCC (2007). Contribution of working group i to the fourth assessment report of the intergovernmental panel on climate change, Climate Change 2007: The Physical Science Basis. United Kingdom y U.S.A. Cambridge University Press.

Itkonen, J. (2010). Internal validity of estimating the carbon kuznets curve by controlling for energy use. IFO Working Paper 95, Institute for Economic Research: University of Munich.

Johnston, J., \& Dinardo, J. (2001). Métodos de econometría. España: Vicens Vives. Kao, C., \& Chiang, M.-H. (2000). On the estimation and inference of a cointegrated regression in panel data. Nonstationary Panels, Panel Cointegration and Dynamic Panels, 15, 179-222.

Kao, C. (1999). Spurious regression and residual based tests for cointegration in panel data. Journal of Econometrics, 90, 1-44.

Kauffman, R., Davidsdottir, B., Garnham, S., \& Pauly, P. (1998). The determinants of atmospheric $\mathrm{SO}_{2}$ Concentrations: Reconsidering the environmental Kuznets curve. Ecological Economics 25, 209-220.

Kuznets, S. (1965). Economic Growth and Structural Change. Nueva York: Norton. Kuznets, S. (1955). Economic growth and income inequality. American Economic Review, 45(1), 1-28.

Kolstad, C.D. (2001). Economía Ambiental. México: Oxford University Press.

Labandeira, X., León, C., \& Vázquez, M.X. (2007). Economía ambiental. España: Pearson-Prentice Hall.

Lee, C.-C. (2005). Energy consumption and GDP in developing countries: A cointegrated panel analysis. Energy Economics, 27, 415-427.

Lee, C.-C., \& Lee, J.-D. (2009). Income and $\mathrm{CO}_{2}$ emissions: Evidence from panel unit root and cointegration tests. Energy Policy, 37, 413-423.

Levine, A., Lin, C.F., \& Chu, C. (2002). Unit root tests in panel data: Asymptotic and finite-sample proprieties. Journal of Econometrics, 108, 1-24.

Maddison, D. (2006). Environmental Kuznets curves: A spatial econometric approach. Journal of Environmental Economics and Management, 51, 218-230.

Meadows, D.H., Meadows, D.L., Randers, J., \& Behrens, W. (1972). The Limits to Growth. Nueva York: Universe Books.

Molina, L., \& Molina, M. (2005). La calidad del aire en la megaciudad de México. Un enfoque integral. México: Fondo de Cultura Económica.

Munasinghe, M. (2002). The sustainomics trans-disciplinary meta-framework for making development more sustainable: Applications to energy issues. International Journal of Sustainable Development, 5, 125-182. 
Munasinghe, M. (1999). Is Environmental degradation an inevitable consequence of economic growth: Tunneling through the environmental Kuznets curve. Ecological Economics, 29, 89-109.

Najam, A., \& Cleveland, C.J. (2003). Energy and sustainable development at global environmental summits: An evolving agenda. Environmental, Development and Sustainability, 5, 117-138.

Narayan, P.K. (2004). Are output fluctuations transitory? New evidence from 24 chinese provinces. Pacific Economic Review, 9(4), 327-336.

Özokcu, S., \& Özdemir, Ö (2017). Economic growth, energy, and environmental Kuznets curve. Renewable and Sustainable Energy Reviews, 72, 639-647.

omc, \& pnuma (2009). El Comercio y el Cambio Climático. Suiza: Organización Mundial de Comercio.

Panayotou, T. (1993). Empirical tests and policy analysis of environmental degradation at different stages of economic development. Working Paper WEP2-22/WP238, Technology and employment programme, international labour office: Geneva.

Pedroni, P. (2004). Panel cointegration: Asymptotic and finite sample properties of pulled time series tests with an application to the PpP hypothesis. New results. Econometric Theory, 20, 597-627.

Pedroni, P. (2000). Fully modified ols for heterogeneous cointegrated panels. Advanced in Econometrics, 15, 93-130.

Pedroni, P. (1999). Critical values for cointegration test in heterogeneous panels with multiple regressors. Oxford Bulletin of Economics and Statistics Special, Issue 0305-9049, 653-670.

Phillips, P. C. B., \& Moon, H. R. (1999). Linear regression limits theory for non-stationary panel data. Econometrica, 67, 1057-1111.

Phillips, P.C.B., \& Hansen, B.E. (1990). Statistical Inference in instrumental variables regression with I(1) processes. Review of Economic Studies, 57(1), 99-125.

Phillips, P. C. B., \& Perron, P. (1988). Testing for a unit root in time series regression. Biometrika, 75, 335-346.

Pindyck, R., \& Rubinfeld, D. (2001). Econometría. Modelos y Pronósticos. México: McGraw-Hill.

PNuma, \& Cepal (2010). Gráficos Vitales del Cambio Climático en América Latina y el Caribe. Santiago de Chile: Santiago de Chile.

Sadorsky, P. (2009). Renewable energy consumption, $\mathrm{CO}_{2}$ emissions and oil prices in the G7 countries. Energy Economics, 31, 456-462.

Sánchez, L., \& Reyes, O. (2015). Medidas de adaptación y mitigación frente al cambio climático en América Latina y el Caribe. Una revisión general. Estudios del Cambio Climático en América Latina. Santiago de Chile: CEPAL. 
Sánchez, L. (2013). Identificación de los patrones de consumo en América Latina. Documento de Trabajo. Santiago de Chile: CEPAL.

Selden, T., \& Song, D. (1994). Environmental quality and development: Is there a Kuznets curve for air pollution? Journal of Environmental Economics and Environmental Management, 27, 147-162.

Shahbaz, M.A., Solarin, S.A., \& Ozturk, I. (2016). Environmental Kuznets curve hypothesis and the role of globalization in selected african countries. Ecological Indicators, 67, 623-636.

Shahbaz, M.A. (2009). Reassessment of finance-growth nexus for Pakistan: Under the investigation of FMOLS and DoLs techniques. The Icfai Journal of Applied Economics, 8(1), 65-81.

Sheinbaum, C., \& Ruiz, B. (2012). Energy context in Latin America. Energy, 40, 39-46.

Simioni, D. (2003). Contaminación atmosférica y conciencia ciudadana. Documento de Trabajo. Santiago de Chile: cepal.

Sims, C.A. (1972). Money, income and causality. American Economic Review, $62,540-552$.

Sinn, H.-W. (2012). The Green Paradox. A Supply-Side Approach to Global Warming. Estados Unidos: The miт Press.

Stern, D. I., \& Cleveland, C. J. (2003). Energy and economic growth. En D.I. Stern, \& C. J. Cleveland (eds.), Encyclopedia of Energy, (pp. 35-51). San Diego: Academic Press.

Stern, D., \& Common, M. (2001). Is there an environmental Kuznets curve for sulfur? Journal of Environmental Economics and Management, 41, 162-178.

Suri, V., \& Chapman, D. (1998). Economic growth, trade and energy: Implications for the environmental Kuznets curve. Ecological Economics, 25, 195-208.

$\mathrm{Xu}, \mathrm{B}$. (2012). Climate change and mitigation in China (tesis doctoral). School of Industrial Engineering and Management, Royal Institute of Techno$\operatorname{logy}($ Ктн): Stockholm.

Yang, G., Sun, T., Wang, J., \& Li, X. (2015). Modeling the nexus between carbon dioxide emissions and economic growth. Energy Policy, 86, 164-177.

Yin, J., Zheng, M., \& Chen, J. (2015). The effects of environmental regulation and technical progress on $\mathrm{CO}_{2}$ Kuznets curve: An evidence from China. Energy Policy, 77, 97-108.

Zilio, M. y, \& Caraballo, M.A. (2014). ¿El final de la curva de Kuznets de carbono? Un análisis semiparamétrico para América Latina y el Caribe. El Trimestre Económico LXXXI, (321), 241-270. 